\title{
Different choices of scaling in homogenisation of diffusion and interfacial exchange in a porous medium
}

\author{
Malte A. Peter*, $\dagger \quad$ Michael Böhm \\ Centre for Industrial Mathematics, FB 3, University of Bremen, Postfach 330 440, \\ 28334 Bremen, Germany
}

\begin{abstract}
Several choices of scaling are investigated for a coupled system of parabolic partial differential equations in a two-phase medium at the microscopic scale. This system may be regarded as modelling a reaction-diffusion problem, the Stokes problem of single-phase flow of a slightly compressible fluid or as a heat conduction problem (with or without interfacial resistance), for example. It is shown that, starting with the same problem on the microscopic scale, different choices of scaling of the diffusion coefficients (resp. permeability or conductivity) and the interfacial-exchange coefficient lead to different types of macroscopic systems of equations. The characterisation of the limit problems in terms of the scaling parameters constitutes a modelling tool because it allows to determine the right type of limit problem. New macroscopic models, not previously dealt with, arise and, for some scalings, classical macroscopic models are recovered. Using the method of two-scale convergence, a unified approach yielding rigorous proofs is given covering a very broad class of different scalings.
\end{abstract}

Keywords: Homogenization, reaction-diffusion equations, multiscale, boundary value problems for parabolic systems, porous media

\section{Introduction}

In this paper, homogenisation of a coupled system of two partial differential equations is investigated, i.e. we are interested in obtaining the system of equations describing the effective behaviour at the macroscopic scale. The specific setting is such that we are looking at a two-phase medium (a porous medium or a two-phase alloy, e.g.) made up of two distinct parts (solid matrix and voids, e.g.) and each equation describes the behaviour of an unknown in one part of the medium. The

\footnotetext{
${ }^{*}$ Correspondence to: M. A. Peter, Department of Mathematics, University of Auckland, Private Bag 92019, Auckland 1142, New Zealand (current address).

${ }^{\dagger}$ E-Mail: mpeter@math.auckland.ac.nz
} 
two equations are coupled by an exchange across the internal boundary separating the two parts. The system of equations we are considering can be used to model different physical processes such as a reaction-diffusion problem, the Stokes problem of single-phase flow of a slightly compressible fluid or as a heat conduction problem (with or without interfacial resistance) amongst others.

The idea of periodic homogenisation is to assume the heterogeneous material to be periodic with respect to a reference cell scaled by a (small) scale parameter. The macroscopic limit problem is then obtained by letting this scale parameter tend to zero. To a certain extent, the periodicity assumption can be relaxed (cf. [1, 2]) and the reference cell may change with time (cf. [3]) but this is not the focus of this work. Instead, the objective of this paper is to investigate the influence of different choices of scaling of the coefficients of the partial differential equations with powers of the scale parameter on the corresponding macroscopic limit problem. It turns out that depending on the choice of these powers, different types of macroscopic problems are obtained in the limit. Usually, it is somewhat up to the modelling of the specific problem which choice (or range) of scaling exponents is appropriate (cf. $[4,5,6]$, e.g.). It can be motivated by a non-dimensionalisation (cf. [7, 2]) or by geometric arguments (cf. [8], e.g.). Hence, the characterisation of the types of limit problems depending on the scaling exponents an also be considered as a modelling tool because it suggests which are the correct (i.e. process-adapted) macroscopic problems.

To be more specific, we consider an open bounded material body $\Omega \subset \mathbb{R}^{n}$, $n \geq 2$, with Lipschitz-continuous boundary, which is a mixture of two different phases. The part of $\Omega$ made up of the first material is denoted by $\Omega^{\mathrm{p}}$ while the other part is labelled with $\Omega^{\mathrm{s}}$. To fix ideas, $\Omega$ is assumed to be a porous medium (motivating the superscripts $\mathrm{p}$ and $\mathrm{s}$ ) although any two-phase composite material can be imagined. It is assumed that $\Omega$ is periodic with respect to a scaled representative cell $Y=(0,1)^{n}$, which contains a solid particle, $Z^{\mathrm{s}}$ and void (pore) space $Z^{\mathrm{p}}$ (each being an open bounded domain with Lipschitz-continuous boundary), i.e. $\Omega$ is the union of a finite amount of translated versions of $\varepsilon Y$. Then, $\Omega_{\varepsilon}^{\alpha}=\Omega \cap \operatorname{int} \bigcup_{k} \varepsilon \bar{Z}_{k}^{\alpha}$, $\alpha \in\{\mathrm{p}, \mathrm{s}\}$, where the subscript $k$ denotes translation of the set by $k \in \mathbb{Z}^{n}$ and $\varepsilon$ indicates the $\varepsilon$-periodic geometry of the domain. It can be noted that $Z^{\mathrm{s}}$ may or may not be completely contained in $Y$ so that $\Omega_{\varepsilon}^{\mathrm{s}}$ may either be connected or not. However, $\Omega_{\varepsilon}^{\mathrm{p}}$ is assumed connected and, for $n=2$, this implies that $\Omega_{\varepsilon}^{\mathrm{s}}$ may not be connected. Moreover, we assume that the representative cell is periodic, by which we mean that opposite faces of the cell are identical. The characteristic function of $Z^{\alpha}$ is denoted by $\chi^{\alpha}: Y \rightarrow\{0,1\}, \alpha \in\{\mathrm{p}, \mathrm{s}\}$, and we write $\chi_{\varepsilon}^{\alpha}(x)=\chi^{\alpha}(x / \varepsilon)$ where $\chi^{\alpha}$ has been extended periodically. The interface between $\Omega_{\varepsilon}^{\mathrm{p}}$ and $\Omega_{\varepsilon}^{\mathrm{s}}$ (internal to $\Omega)$ is denoted by $\Gamma_{\varepsilon}$ and the considered time interval is labelled with $S=(0, T)$. The idea of periodic homogenisation (cf. $[9,10]$, e.g.) is then to examine the limit as $\varepsilon$ approaches zero in order to obtain averaged problems defined in all of $\Omega$ which are easier to treat numerically and give useful information about macroscopically observable processes.

We consider the problem of diffusion (and reaction) of a species in the two parts of the porous medium. Denoting its mass concentration by $u$, the diffusion and reaction of the species in the pores and the solid matrix as well as an exchange 
between the two phases can be described by

$$
\begin{aligned}
& \partial_{t} u_{\varepsilon}^{\mathrm{p}}(x, t)-\nabla \cdot\left(\varepsilon^{k} D_{\varepsilon}^{\mathrm{p}} \nabla u_{\varepsilon}^{\mathrm{p}}\right)=f_{\varepsilon}^{\mathrm{p}}, \quad x \in \Omega_{\varepsilon}^{\mathrm{p}}, t \in S, \\
& \partial_{t} u_{\varepsilon}^{\mathrm{s}}(x, t)-\nabla \cdot\left(\varepsilon^{l} D_{\varepsilon}^{\mathrm{s}} \nabla u_{\varepsilon}^{\mathrm{s}}\right)=f_{\varepsilon}^{\mathrm{s}}, \quad x \in \Omega_{\varepsilon}^{\mathrm{s}}, t \in S, \\
& -\left(\varepsilon^{k} D_{\varepsilon}^{\mathrm{p}} \nabla u_{\varepsilon}^{\mathrm{p}}\right) \cdot \nu_{\varepsilon}^{\mathrm{p}}=\left(\varepsilon^{l} D_{\varepsilon}^{\mathrm{s}} \nabla u_{\varepsilon}^{\mathrm{s}}\right) \cdot \nu_{\varepsilon}^{\mathrm{s}}, \quad x \in \Gamma_{\varepsilon}, t \in S, \\
& -\left(\varepsilon^{k} D_{\varepsilon}^{\mathrm{p}} \nabla u_{\varepsilon}^{\mathrm{p}}\right) \cdot \nu_{\varepsilon}^{\mathrm{p}}=\varepsilon^{m} a_{\varepsilon}\left(u_{\varepsilon}^{\mathrm{p}}-u_{\varepsilon}^{\mathrm{s}}\right), \quad x \in \Gamma_{\varepsilon}, t \in S .
\end{aligned}
$$

The scaling exponents $k, l$ and $m$ are real numbers and $k, l \geq 0$. Owing to the symmetry of the problem, we assume $k<l$ if $k \neq l$ without restriction of generality. The values of the scaling exponents can be motivated by a non-dimensionalisation, e.g. According to [7], the values of the numbers $k, l$ and $m$ are related to the characteristic times of diffusion associated with $u_{\varepsilon}^{\mathrm{p}}$ and $u_{\varepsilon}^{\mathrm{s}}$ and the speed of the interfacial exchange, respectively, as well as the characteristic macroscopic and microscopic length scales.

Let

$$
\begin{gathered}
\mathcal{V}(\Omega)=L^{2}\left(0, T ; W^{1,2}(\Omega)\right), \quad \mathscr{V}(\Omega)=\left\{u \in \mathcal{V}(\Omega) \mid \partial_{t} u \in L^{2}(\Omega \times(0, T))\right\}, \\
(u(t) \mid v(t))_{\Omega}=\int_{\Omega} u(x, t) v(x, t) \mathrm{d} x, \quad(u \mid v)_{\Omega, t}=\int_{0}^{t}(u(t) \mid v(t))_{\Omega} \mathrm{d} t
\end{gathered}
$$

$|u(t)|_{\Omega}^{2}=(u(t) \mid u(t))_{\Omega}$ and $|u|_{\Omega, t}^{2}=(u \mid u)_{\Omega, t}$. The (standard) weak form of problem (1) reads as follows: Find $\left(u_{\varepsilon}^{\mathrm{p}}, u_{\varepsilon}^{\mathrm{s}}\right) \in \mathcal{V}\left(\Omega_{\varepsilon}^{\mathrm{p}}\right) \times \mathcal{V}\left(\Omega_{\varepsilon}^{\mathrm{p}}\right)$ such that $\left(u_{\varepsilon}^{\mathrm{p}}(0), u_{\varepsilon}^{\mathrm{s}}(0)\right)=$ $\left(u_{0}^{\mathrm{p}}, u_{0}^{\mathrm{s}}\right) \in\left[L^{2}(\Omega)\right]^{2}$ and

$$
\begin{gathered}
\left(\partial_{t} u_{\varepsilon}^{\mathrm{p}}(t) \mid \phi(t)\right)_{\Omega_{\varepsilon}^{\mathrm{p}}}+\varepsilon^{k}\left(D_{\varepsilon}^{\mathrm{p}}(t) \nabla u_{\varepsilon}^{\mathrm{p}}(t) \mid \nabla \phi(t)\right)_{\Omega_{\varepsilon}^{\mathrm{p}}} \\
\quad=\left(f_{\varepsilon}^{\mathrm{p}}(t) \mid \phi(t)\right)_{\Omega_{\varepsilon}^{\mathrm{p}}}-\varepsilon^{m}\left(f_{\varepsilon}^{\mathrm{ex}}(t) \mid \phi(t)\right)_{\Gamma_{\varepsilon}} \\
\begin{aligned}
\left(\partial_{t} u_{\varepsilon}^{\mathrm{s}}(t) \mid \psi(t)\right)_{\Omega_{\varepsilon}^{\mathrm{s}}}+\varepsilon^{l}\left(D_{\varepsilon}^{\mathrm{s}}(t) \nabla u_{\varepsilon}^{\mathrm{s}}(t) \mid \nabla\right. & \psi(t))_{\Omega_{\varepsilon}^{\mathrm{s}}} \\
& =\left(f_{\varepsilon}^{\mathrm{s}}(t) \mid \psi(t)\right)_{\Omega_{\varepsilon}^{\mathrm{s}}}+\varepsilon^{m}\left(f_{\varepsilon}^{\mathrm{ex}}(t) \mid \psi(t)\right)_{\Gamma_{\varepsilon}}
\end{aligned}
\end{gathered}
$$

for all $(\phi, \psi) \in \mathcal{V}\left(\Omega_{\varepsilon}^{\mathrm{p}}\right) \times \mathcal{V}\left(\Omega_{\varepsilon}^{\mathrm{s}}\right)$ and a.e. $t \in S$.

Depending on the choice of boundary conditions at the exterior boundary of $\Omega$, additional terms may appear in (4a) and (4b) or the space $\mathcal{V}$ needs to be chosen slightly differently. Different reasonable options are possible, but as these do not pose problems in the homogenisation process (other than of technical nature), they are not explicitly included in the formulation of the problem. For simplicity, we assume homogeneous Neumann conditions at all external boundaries.

For the coefficients of the system, we assume that their space variables can each be split into a macroscopic and a microscopic one (often called slow and fast component): We assume that there exist functions $D^{\alpha}=D^{\alpha}(x, y, t), x \in \Omega_{\varepsilon}^{\alpha}, y \in$ $Z^{\alpha}, t \in S$, bounded from above and away from zero and periodically extended in $y$, such that $D_{\varepsilon}^{\alpha}=D^{\alpha}(x, x / \varepsilon, t), \alpha \in\{\mathrm{p}, \mathrm{s}\}$, and, analogously, for $a_{\varepsilon}$ and $f_{\varepsilon}^{\alpha}, \alpha \in\{\mathrm{p}, \mathrm{s}\}$. These function are extended to all of $\Omega$ by zero. We further assume

$$
\lim _{\varepsilon \rightarrow 0}\left|D_{\varepsilon}^{\alpha}(t)\right|_{\Omega}^{2}=\left|D^{\alpha}(t)\right|_{\Omega \times Y}^{2}, \quad \lim _{\varepsilon \rightarrow 0} \varepsilon\left|a_{\varepsilon}(t)\right|_{\Gamma_{\varepsilon}}^{2}=|a(t)|_{\Omega \times \Gamma}^{2} .
$$

At this point, it should be noted that several classical homogenisation problems can be recovered from this system. For example, the distributed-microstructure 
model of [8] modelling the flow of a slightly compressible fluid in a fissured medium is obtained for $k=0, l=2, m=0$. The parallel-flow model for basically the same physical application (the macromodel of which has been suggested by [11]) is obtained for $k=0, l=0, m=1$ (cf. [12] for the stationary case). The problem of miscible displacement in a porous medium has been considered by [13] where also a Robin-type transmission condition at the pore-matrix interface is assumed. Here, the choice of scaling was $k=0, l=2, m=1$. A similar problem was also considered by [14], where the $u_{\varepsilon}^{\mathrm{s}}$ is a surface concentration on $\Gamma_{\varepsilon}$. Numerous other examples of special cases of problem (1) can be found in the literature where convergence has been proven or the macroscopic equations where formally derived by asymptotic expansion, cf. $[1,15,16]$ and references given therein. We finally would like to mention [17], where $k=0, m=1$ and an ordinary differential equation for $u_{\varepsilon}^{\mathrm{s}}$ is assumed but the exchange term may be more general, that is monotone or a maximal-monotone graph, and the work of [18], who considered the problem with $k=0, l=2, m=1$ and a rather general exchange term as well as certain concentration-dependent reaction rates.

Moreover, similar problems have been investigated for varying choices of scaling. A related problem is discussed in [19] where a matched boundary condition is assumed at the pore-matrix interface and $k=0$ is fixed but different choices of $l$ are distinguished. It must also be noted that in these considerations, $\Omega_{\varepsilon}^{\mathrm{s}}$ is assumed disconnected. For $k=l=0$ and varying $m$, similar problems have been studied quite extensively. For example, [4] seem to be the first to examine different choices of $m$ by formal techniques. Most notably, [20] (rigorously) discuss the stationary version of problem (1) with $k=l=0$ varying $m$. It is also worth mentioning [21] who examines (but does not rigorously prove) the case $k=l=0$ for different choices of $m$ as well as [22], [23] and [24] who particularly investigate the case of $\Omega_{\varepsilon}^{\mathrm{s}}$ being disconnected. Moreover, [25] rigorously treats the case where $\Omega_{\varepsilon}^{\mathrm{p}}$ and $\Omega_{\varepsilon}^{\mathrm{s}}$ are separated by a (three-dimensional) layer and [6] discuss different choices of $l$ and $m$ where $k=0$ by formal techniques for a similar problem. Finally, in the monograph [26], the influence of certain different scalings in porous-media-flow problems is extensively discussed from a more applied point of view.

Therefore, the homogenisation results for (4) found in the literature are limited to $k=l=0$ and $m$ arbitrary as well as $k=0, l=2, m=1$. New scalings considered in this work particularly include $k \geq 0, l>0$, and $m$ arbitrary. The most interesting new limit problems arise for $k=0, m=-1$ and different values of $l \geq 0$.

In what follows, the convergence of problem (4) is examined using two-scale convergence yielding a unified and mathematically rigorous way of obtaining the associated macroscopic limit problems depending on the choice of scaling exponents. First, the macroscopic limit problems associated with problem (4) are stated depending on $k, l$ and $m$ in $\S 2$. Classical results found in the literature are recovered and new macroscopic problems are obtained. In §3, a-priori estimates for the solutions are proven and the convergence of the sequences of solutions is discussed in the context of two-scale convergence. Classical results are briefly summarised, where the distinction of $\Omega_{\varepsilon}^{\mathrm{s}}$ being connected or disconnected is of relevance, and new results are presented concerning the two-scale convergence of the interfacial-exchange term. In $\S 4$, these results are used to determine the limit problems (stated in $\S 2$ ). 


\section{The macroscopic limit problems - summary of the results}

The macroscopic limit problems of problem (4) are now stated. We denote the limit functions of $u_{\varepsilon}^{\mathrm{p}}$ and $u_{\varepsilon}^{\mathrm{s}}$ as $\varepsilon \rightarrow 0$ by $u^{\mathrm{p}}$ and $u^{\mathrm{s}}$, respectively. Obviously, different choices of the scaling exponents $k, l$ and $m$ need to be distinguished. In $\S 4$, the convergence is rigorously proven.

To simplify notation, the superscript $\alpha$ is introduced which, if not stated otherwise, stands for $\mathrm{p}$ and $\mathrm{s}$, i.e. statements about $D^{\alpha}$ apply to $D^{\mathrm{p}}$ and $D^{\mathrm{s}}$, e.g. Furthermore, when referring to $u^{\alpha}$, the scaling exponent is denoted by $\lambda$, i.e. $\lambda=k$ if $\alpha=\mathrm{p}$ and $\lambda=l$ if $\alpha=\mathrm{s}$. The limits of the right-hand sides, $f_{\varepsilon}^{\alpha}$, are denoted by $f^{\alpha}$.

In order to be able to write the macroscopic limit equations in a simple way, two factors are introduced:

$$
\theta(\lambda)=\left\{\begin{array}{ll}
1, & \lambda=0 \text { or } \lambda=2, \\
0, & 0<\lambda<2 \text { or } \lambda>2,
\end{array} \quad \sigma^{\alpha}(m)= \begin{cases}-1, & \alpha=\mathrm{p}, m=1 \\
1, & \alpha=\mathrm{s}, m=1 \\
0, & \alpha \in\{\mathrm{p}, \mathrm{s}\}, m \neq 1\end{cases}\right.
$$

It is useful to distinguish the cases $m<1$ and $m \geq 1$ as these correspond to particularly different limit behaviours. It turns out that independently of the choice of $m, u^{\alpha}$ is independent of $y$ if $\lambda<2$. Moreover, the homogeneous Neumann boundary conditions on the external boundary of $\Omega$ are recovered. This is also formally true for the case that $\partial \Omega_{\varepsilon}^{\alpha} \cap \partial \Omega=\emptyset$. After the statements of the limit problems in $\S 2.1$ (for $m<1$ ) and $\S 2.2$ (for $m \geq 1$ ), a qualitative summary is given in $\S 2.3$.

\subsection{The case $m<1$}

We begin with the case $m<1$. All of the following results, except for the special case $k=l=0$, seem to be new. For $m<1$, it turns out that the limit functions satisfy $u^{\mathrm{p}}(x, y, t)=u^{\mathrm{s}}(x, y, t)$ for a.e. $x \in \Omega, y \in \Gamma, t \in S$. It therefore makes sense to define

$$
u(x, y, t)=\chi^{\mathrm{p}}(y) u^{\mathrm{p}}(x, y, t)+\chi^{\mathrm{s}}(y) u^{\mathrm{s}}(x, y, t)
$$

and to look for the one equation satisfied by $u$. For ease of notation, we also define

$$
f(x, y, t)=\chi^{\mathrm{p}}(y) f^{\mathrm{p}}(x, y, t)+\chi^{\mathrm{s}}(y) f^{\mathrm{s}}(x, y, t)
$$

and, analogously, $D$.

We require the solutions of the following cell problem: Let $\varsigma_{j}^{m, l}, j=1, \ldots, n$, be the $Y$-periodic solution of the cell problem

$$
-\nabla_{y} \cdot\left(D(x, y, t)\left(\nabla_{y} \varsigma_{j}^{m, l}(x, y, t)+e_{j}\right)\right)=0, \quad y \in X, x \in \Omega, t \in S,
$$


where, if $l=0, X=Z^{\mathrm{p}} \cup Z^{\mathrm{s}}$ subject to the following boundary conditions on $\Gamma$,

$$
\begin{aligned}
& -D^{\alpha}\left(\nabla_{y}\left(\varsigma_{j}^{m, l}\right)^{\alpha}+e_{j}\right) \cdot \nu^{\alpha}=0, \quad \text { if } m>-1, \\
& -D^{\alpha}\left(\nabla_{y}\left(\varsigma_{j}^{m, l}\right)^{\alpha}+e_{j}\right) \cdot \nu^{\alpha}+\sigma^{\alpha}(1) a\left[\left(\varsigma_{j}^{m, l}\right)^{\mathrm{p}}-\left(\varsigma_{j}^{m, l}\right)^{\mathrm{s}}\right]=0, \quad \text { if } m=-1 \text {, } \\
& \left.\begin{array}{rl}
\left(\varsigma_{j}^{m, l}\right)^{\mathrm{p}}-\left(\varsigma_{j}^{m, l}\right)^{\mathrm{s}} & =0, \\
-D^{\mathrm{p}}\left(\nabla_{y}\left(\varsigma_{j}^{m, l}\right)^{\mathrm{p}}+e_{j}\right) \cdot \nu^{\mathrm{p}}-D^{\mathrm{s}}\left(\nabla_{y}\left(\varsigma_{j}^{m, l}\right)^{\mathrm{s}}+e_{j}\right) \cdot \nu^{\mathrm{s}}=0,
\end{array}\right\} \quad \text { if } m<-1,
\end{aligned}
$$

and, if $l>0, X=Z^{\mathrm{p}}$ subject to the following boundary conditions on $\Gamma$,

$$
\begin{array}{rlrl}
-D^{\mathrm{p}}\left(\nabla_{y} \varsigma_{j}^{m, l}+e_{j}\right) \cdot \nu^{\alpha} & =0, & & \text { if } m>-1, \\
-D^{\mathrm{p}}\left(\nabla_{y} \varsigma_{j}^{m, l}+e_{j}\right) \cdot \nu^{\mathrm{p}}+a\left(\varsigma_{j}^{m, l}+y \cdot \nabla u\right)=0, & & \text { if } m=-1, \\
\varsigma_{j}^{m, l}=0, & & \text { if } m<-1 .
\end{array}
$$

This allows the definition of the tensors $P^{m, l}=\left[p_{i j}^{m, l}\right]_{i j}$ via

$$
p_{i j}^{m, l}(x, t)=\int_{Y} D(x, y, t)\left(\delta_{i j}+\partial_{y_{i}} \varsigma_{j}^{m, l}(x, y, t)\right) \mathrm{d} y,
$$

Note that in the case $l>0$ and $m=-1$, this tensor is dependent on $\nabla u$.

The limit problems can now be stated (where an additional assumption is necessary if $k \neq l$ and $m \leq k-1$, cf. (40)):

If $0 \leq k, l<2$, the macroscopic limit problem of problem (4) is given by

$$
\left(\partial_{t} u(t) \mid \phi(t)\right)_{\Omega}+\theta(k)\left(P^{m, l}(t) \nabla u(t) \mid \nabla \phi(t)\right)_{\Omega}=\left(\int_{Y} f(\cdot, y, t) \mathrm{d} y \mid \phi(t)\right)_{\Omega}
$$

for all $\phi \in \mathcal{V}(\Omega)$ and a.e. $t \in S$.

If $k, l \geq 2$, the macroscopic limit problem is given by

$$
\begin{aligned}
\left(\partial_{t} u(t) \mid \psi(t)\right)_{\Omega \times Y}+\theta(k) & \left(D(t) \nabla_{y} u(t) \mid \nabla_{y} \psi(t)\right)_{\Omega \times Z^{\mathrm{p}}} \\
& +\theta(l)\left(D(t) \nabla_{y} u(t) \mid \nabla_{y} \psi(t)\right)_{\Omega \times Z^{\mathrm{s}}}=(f(t) \mid \psi(t))_{\Omega \times Y}
\end{aligned}
$$

for all $\psi \in \mathcal{W}(Y)$ and a.e. $t \in S$ where $\psi^{\mathrm{p}}=\psi^{\mathrm{s}}$ on $\Gamma$ and, moreover, $\psi^{\mathrm{p}}=0$ on $\Gamma$ if $k \neq l$ (this is the case if there is a diffusion term; if not, the corresponding test function only has to be in $\left.L^{2}\left(\Omega \times Z^{\alpha} \times(0, T)\right)\right)$. The space $\mathcal{W}(\cdot)$ is defined as

$$
\left.\mathcal{W}(Y)=L^{2}\left((0, T) \times \Omega ; W_{\#}^{1,2}(Y)\right)\right)
$$

where the subscript \# denotes periodicity.

If $k<2$ and $l \geq 2$ and we have an additional estimate of the type (54) (cf. the discussion in $\S 4$ ), the limit problem is given by

$$
\begin{aligned}
& \left|Z^{\mathrm{p}}\right|\left(\partial_{t} u^{\mathrm{p}}(t) \mid \phi(t)\right)_{\Omega}+\theta(k)\left(P^{m, l}(t) \nabla u^{\mathrm{p}}(t) \mid \nabla \phi(t)\right)_{\Omega} \\
& \quad=-\theta(l)\left(\int_{\Gamma} D^{\mathrm{s}}(\cdot, y, t) \nabla_{y} u^{\mathrm{s}}(\cdot, y, t) \cdot \nu^{\mathrm{s}} \mathrm{d} \sigma_{y} \mid \phi(t)\right)_{\Omega}+\left(\int_{Z^{\mathrm{p}}} f^{\mathrm{p}}(\cdot, y, t) \mathrm{d} y \mid \phi(t)\right)_{\Omega} \\
& \left(\partial_{t} u^{\mathrm{s}}(t) \mid \psi(t)\right)_{\Omega \times Z^{\mathrm{s}}}+\theta(l)\left(D^{\mathrm{s}}(t) \nabla_{y} u^{\mathrm{s}}(t) \mid \nabla_{y} \psi(t)\right)_{\Omega \times Z^{\mathrm{s}}}=\left(f^{\mathrm{s}}(t) \mid \psi(t)\right)_{\Omega \times Z^{\mathrm{s}}}
\end{aligned}
$$

for all $(\phi, \psi) \in \mathcal{V} \times \mathcal{W}\left(Z^{\mathrm{s}}\right)$, where $\psi=0$ on $\Gamma$, and a.e. $t \in S$, together with $u^{\mathrm{s}}=u^{\mathrm{p}}$ on $\Gamma$. Note that the tensor $P^{m, l}$ only appears in the above equations if $k=0$. 


\subsection{The case $m \geq 1$}

If $m \geq 1$, the limit functions $u^{\mathrm{p}}$ and $u^{\mathrm{s}}$ need to be considered separately. Therefore, it suffices to discuss the limit problem associated with $u^{\alpha}$. The total limit problem of (4) is then given by the respective equations for $\alpha=\mathrm{p}$ and $\alpha=\mathrm{s}$. Classical results from the literature are recovered for $k=l=0, m \geq 1$ as well as $k=0, l=2$, $m=1$, while new results are obtained for the other scalings, in particular for $k \geq 0$, $0<l<2, m \geq 1$.

The solutions of two cell problems are required. Let $\varsigma_{j}^{\alpha}, j=1, \ldots, n$, be the $Y$-periodic solution of the cell problem

$$
\begin{array}{cl}
-\nabla_{y} \cdot\left(D^{\alpha}(x, y, t)\left(\nabla_{y} \varsigma_{j}^{\alpha}(x, y, t)+e_{j}\right)\right)=0, & y \in Z^{\alpha}, x \in \Omega, t \in S, \\
-D^{\alpha}(x, y, t)\left(\nabla_{y} \varsigma_{j}^{\alpha}(x, y, t)+e_{j}\right) \cdot \nu^{\alpha}=0, & y \in \Gamma, x \in \Omega, t \in S .
\end{array}
$$

This allows the definition of the tensors $P^{\alpha}=\left[p_{i j}^{\alpha}\right]_{i j}$ via

$$
p_{i j}^{\alpha}(x, t)=\int_{Z^{\alpha}} D^{\alpha}(x, y, t)\left(\delta_{i j}+\partial_{y_{i}} \varsigma_{j}^{\alpha}(x, y, t)\right) \mathrm{d} y .
$$

In order to be able to write the macroscopic limit equations in a simple way, the limit of the interfacial-exchange term is written as

$$
f^{\mathrm{ex}}(x, y, t)=a(x, y, t)\left(u^{\mathrm{p}}(x, y, t)-u^{\mathrm{s}}(x, y, t)\right) .
$$

The limit problems can now be stated where $m>1$ is required if $l>2$ :

If $0 \leq \lambda<2$, the limit function is of the form $u^{\alpha}=u^{\alpha}(x, t)$ and the macroscopic limit problem is given by

$$
\begin{aligned}
\left|Z^{\alpha}\right|\left(\partial_{t} u^{\alpha}(t) \mid\right. & \phi(t))_{\Omega}+\theta(\lambda)\left(P^{\alpha}(t) \nabla u^{\alpha}(t) \mid \nabla \phi(t)\right)_{\Omega} \\
& =\left(\int_{Z^{\alpha}} f^{\alpha}(\cdot, y, t) \mathrm{d} y \mid \phi(t)\right)_{\Omega}+\sigma^{\alpha}(m)\left(\int_{\Gamma} f^{\operatorname{ex}}(\cdot, y, t) \mathrm{d} \sigma_{y} \mid \phi(t)\right)_{\Omega}
\end{aligned}
$$

for all $\phi \in \mathcal{V}(\Omega)$ and a.e. $t \in S$.

If $\lambda \geq 2$, the limit function is of the form $u^{\alpha}=u^{\alpha}(x, y, t)$ and the macroscopic limit problem is given by

$$
\begin{aligned}
\left(\partial_{t} u^{\alpha}(t) \mid \psi(t)\right)_{\Omega \times Z^{\alpha}}+\theta(\lambda)\left(D^{\alpha}(t) \nabla_{y} u^{\alpha}(t) \mid \nabla_{y} \psi(t)\right)_{\Omega \times Z^{\alpha}} & \\
& =\left(f^{\alpha}(t) \mid \psi(t)\right)_{\Omega \times Z^{\alpha}}+\sigma^{\alpha}(m)\left(f^{\operatorname{ex}}(t) \mid \psi(t)\right)_{\Omega \times \Gamma}
\end{aligned}
$$

for all $\psi \in \mathcal{W}\left(Z^{\alpha}\right)$ and a.e. $t \in S$.

\subsection{Qualitative summary}

Qualitatively, we have the following limit behaviour depending on the scaling of the diffusion term of the corresponding species:

- $\lambda=0$ : macroscopic diffusion.

- $\lambda=2$ : microscopic diffusion. 
- $0<\lambda<2$ or $\lambda>2$ : no diffusion.

The limit behaviour of the interfacial-exchange term can be summarised as follows:

- $m>$ 1: no interfacial exchange.

- $m=1$ : interfacial exchange is governed by a macroscopic volume term; no microscopic interfacial exchange.

- $-1<m<1$ : infinitely fast interfacial exchange on the macroscopic scale; no microscopic interfacial exchange.

- $m=-1$ : infinitely fast interfacial exchange on the macroscopic scale; microscopic interfacial exchange.

- $m<-1$ : infinitely fast interfacial exchange on both the macroscopic and the microscopic scale.

\section{Convergence of the micro-solutions}

In this section, $\varepsilon$-independent a-priori estimates for the solutions of the microscopic problems are obtained first. Then, the convergence of sequences satisfying such apriori estimates is generally discussed in the context of two-scale convergence. For this purpose, some well-known results are summarised and formulated in the general context considered here and new results required due to the broad range of choices of parameters $k, l$ and $m$ are developed.

\subsection{A-priori estimates}

\section{Theorem 3.1}

For fixed $0<\varepsilon \leq 1$, there exists a solution $\left(u_{\varepsilon}^{\mathrm{p}}, u_{\varepsilon}^{\mathrm{s}}\right) \in \mathscr{V}\left(\Omega_{\varepsilon}^{\mathrm{p}}\right) \times \mathscr{V}\left(\Omega_{\varepsilon}^{\mathrm{s}}\right)$ of problem $(4)$ such that

$$
\left|u_{\varepsilon}^{\mathrm{p}}(t)\right|_{\Omega_{\varepsilon}^{\mathrm{p}}}+\varepsilon^{k / 2}\left|\nabla u_{\varepsilon}^{\mathrm{p}}\right|_{\Omega_{\varepsilon}^{\mathrm{p}}, t}+\left|u_{\varepsilon}^{\mathrm{s}}(t)\right|_{\Omega_{\varepsilon}^{\mathrm{s}}}+\varepsilon^{l / 2}\left|\nabla u_{\varepsilon}^{\mathrm{s}}\right|_{\Omega_{\varepsilon}^{\mathrm{s}}, t}+\varepsilon^{m / 2}\left|u_{\varepsilon}^{\mathrm{p}}-u_{\varepsilon}^{\mathrm{s}}\right|_{\Gamma_{\varepsilon}, t} \leq C
$$

holds for a.e. $t \in S$, where the constant $C$ depends on $T$ and the data but not on $\varepsilon$.

Proof The existence of the solutions follows from standard arguments while the proof of (22) can be obtained from the usual parabolic energy estimates. Care must be taken in order to obtain the estimate for the boundary term. This can be achieved by adding (4a) and (4b) and choosing the test function as

$$
\phi(x, t)= \begin{cases}u_{\varepsilon}^{\mathrm{p}}(x, t), & x \in \Omega_{\varepsilon}^{\mathrm{p}}, t \in(0, T), \\ u_{\varepsilon}^{\mathrm{s}}(x, t), & x \in \Omega_{\varepsilon}^{\mathrm{s}}, t \in(0, T),\end{cases}
$$

Standard procedures then give the result. 


\subsection{Some preparations concerning two-scale convergence}

The convergence of the sequences satisfying a-priori estimates of the type (22) is now discussed in the general context of two-scale convergence. First, a few results on two-scale convergence are cited for convenience and adapted and generalised to the requirements in this context. For details concerning classical results we refer to [27], [28], [29], [30], [31] and [32]. Then, some special results by [20] are extended to the more general case considered here which are required for the examination of the convergence of the interfacial-exchange term. For simplicity, we discuss the twoscale convergence for sequences independent of time. Since time is only a parameter with respect to two-scale convergence, this is no restriction (also cf. remark 3.10).

\section{Definition 3.2 (Two-scale convergence)}

A sequence of functions $v_{\varepsilon}$ in $L^{2}(\Omega)$ is said to two-scale converge to a limit function $v_{0}(x, y) \in L^{2}(\Omega \times Y)$ iff

$$
\lim _{\varepsilon \rightarrow 0} \int_{\Omega} v_{\varepsilon}(x) \phi(x, x / \varepsilon) \mathrm{d} x=\int_{\Omega} \int_{Y} v_{0}(x, y) \phi(x, y) \mathrm{d} y \mathrm{~d} x
$$

for all $\phi \in C_{0}^{\infty}\left(\Omega ; C_{\#}^{\infty}(Y)\right)$ where the subscript \# denotes periodicity. A sequence of functions $v_{\varepsilon}$ in $L^{2}\left(\Gamma_{\varepsilon}\right)$ is said to two-scale converge to a limit function $v_{0}(x, y) \in$ $L^{2}(\Omega \times \Gamma)$ iff

$$
\lim _{\varepsilon \rightarrow 0} \varepsilon \int_{\Gamma_{\varepsilon}} v_{\varepsilon}(x) \phi(x, x / \varepsilon) \mathrm{d} \sigma_{x}=\int_{\Omega} \int_{\Gamma} v_{0}(x, y) \phi(x, y) \mathrm{d} \sigma_{y} \mathrm{~d} x
$$

for all $\phi \in C_{0}^{\infty}\left(\Omega ; C_{\#}^{\infty}(Y)\right)$.

The following two theorems summarise some aspects of two-scale convergence of bounded sequences in $L^{2}\left(\Omega_{\varepsilon}^{\alpha}\right)$ and $W^{1,2}\left(\Omega_{\varepsilon}^{\alpha}\right)$, respectively. These are standard results (cf. [27, 28, 29]) or minor variations of such, which can be obtained analogously (cf. [2]). A special notation is introduced: For a function $v^{\alpha} \in L^{2}\left(\Omega_{\varepsilon}^{\alpha}\right)$, its zero extension to $\Omega$ is denoted by $\tilde{v}^{\alpha}$. Clearly: $\tilde{v}^{\alpha} \in L^{2}(\Omega)$.

We begin with the two-scale convergence of $L^{2}$-bounded sequences.

\section{Theorem 3.3}

(a) Let $v_{\varepsilon}$ be a bounded sequence in $L^{2}(\Omega)$. Then, there exists a subsequence such that $v_{\varepsilon}$ two-scale converges to a limit function $v_{0} \in L^{2}(\Omega \times Y)$. If $v_{\varepsilon}$ is a strongly-convergent sequence in $L^{2}(\Omega)$, it converges to the same limit, which is independent of $y$, in two-scale sense.

(b) If $v_{\varepsilon}$ is a bounded sequence in $L^{2}\left(\Omega_{\varepsilon}^{\alpha}\right)$, the two-scale limit function of $\tilde{v}_{\varepsilon}$ has the form $v_{0}(x, y)=\chi^{\alpha}(y) v_{*}(x, y)$ with $v_{*} \in L^{2}(\Omega \times Y)$, i.e. $v_{0}(x, y)=0$ for $y \in Y \backslash \bar{Z}^{\alpha}$.

(c) For a sequence $v_{\varepsilon}$ in $L^{2}\left(\Gamma_{\varepsilon}\right)$ satisfying the estimate

$$
\varepsilon\left|v_{\varepsilon}\right|_{\Gamma_{\varepsilon}}^{2} \leq C
$$


there exists a two-scale limit $v_{0} \in L^{2}\left(\Omega ; L^{2}(\Gamma)\right)$ such that $v_{\varepsilon}$ two-scale converges to $v_{0}$ in the sense (24) and the following estimate holds

$$
\lim _{\varepsilon \rightarrow 0} \varepsilon \int_{\Gamma_{\varepsilon}}\left|v_{\varepsilon}(x)\right|^{2} \mathrm{~d} x \geq \int_{\Omega} \int_{\Gamma}\left|v_{0}(x, y)\right|^{2} \mathrm{~d} \sigma_{y} \mathrm{~d} x
$$

The two-scale convergence of $W^{1,2}$-bounded sequences is more complicated. Two aspects are important in this context: the scaling exponent in (27) and the connectedness (or disconnectedness) of the domain. The proofs of the parts of the next theorem are all similar (except for that of part (d) which can be found in [29]) and can be obtained by minor modifications of proofs already found in the literature. For example, the proof of part (a) for $\Omega_{\varepsilon}^{\alpha}$ connected, follows from theorem 4.6 in [31]. This theorem is in fact formulated for the more general case of multi-scale convergence and the proof for the special case of two-scale convergence is already implicitly contained in the proof of theorem 2.9 of [28]. The proof of part (b) is also implicitly contained in [28]. Since parts (a), (b) and (d) are very minor extensions of well-known results, we only present the proof of parts (c) and (e), which we believe to have not appeared previously, in detail.

\section{Theorem 3.4}

Let $\lambda \geq 0$ be given and $v_{\varepsilon}$ be a sequence of functions in $W^{1,2}\left(\Omega_{\varepsilon}^{\alpha}\right)$ such that

$$
\left|v_{\varepsilon}\right|_{L^{2}\left(\Omega_{\varepsilon}^{\alpha}\right)}+\varepsilon^{\lambda / 2}\left|\nabla v_{\varepsilon}\right|_{L^{2}\left(\Omega_{\varepsilon}^{\alpha}\right)} \leq C .
$$

Then, the following statements hold:

(a) If $\lambda=0$, there exists $v_{0} \in L^{2}(\Omega)$ and $\xi_{0} \in L^{2}(\Omega \times Y)$ such that, up to a subsequence,

$$
\tilde{u}_{\varepsilon} \longrightarrow \chi^{\alpha}(y) v(x) \quad \text { and } \quad \widetilde{\nabla v}_{\varepsilon} \longrightarrow \chi^{\alpha}(y) \xi_{0}(x, y)
$$

in two-scale sense. If, moreover, $\Omega_{\varepsilon}^{\alpha}$ is connected, the limit function $v_{0}$ belongs to $W^{1,2}(\Omega)$ and the limit of the sequence of gradients has the form $\xi_{0}(x, y)=$ $\nabla_{x} v_{0}(x)+\nabla_{y} v_{1}(x, y)$ with $v_{1} \in L^{2}\left(\Omega ; W_{\#}^{1,2}\left(Z^{\alpha}\right) / \mathbb{R}\right)$.

(b) If $\lambda=2$, there exists a two-scale limit $v_{0} \in L^{2}\left(\Omega ; W_{\#}^{1,2}(Y)\right)$ such that, up to a subsequence,

$$
\tilde{v}_{\varepsilon} \longrightarrow \chi^{\alpha}(y) v_{0}(x, y) \quad \text { and } \quad \varepsilon \widetilde{\nabla v_{\varepsilon}} \longrightarrow \chi^{\alpha}(y) \nabla_{y} v_{0}(x, y) .
$$

(c) If $0<\lambda<2$, the conclusions from part (b) apply and, moreover, the limit function $v_{0}$ is independent of $y$. Also, up to a subsequence, the convergence

$$
\varepsilon^{\lambda / 2} \widetilde{\nabla} v_{\varepsilon} \longrightarrow \chi^{\alpha}(y) \nabla_{y} v_{1}(x, y)
$$

holds in two-scale sense with $v_{1} \in L^{2}\left(\Omega ; W_{\#}^{1,2}\left(Z^{\alpha}\right) / \mathbb{R}\right)$.

(d) In any of the previous cases, the traces of $v_{\varepsilon}$ on $\Gamma_{\varepsilon}$ two-scale converge on $L^{2}\left(\Gamma_{\varepsilon}\right)$ and (26) holds. 
(e) If $\lambda>2$, there exists a two-scale limit $v_{0} \in L^{2}(\Omega \times Y)$ such that, up to a subsequence,

$$
\tilde{v}_{\varepsilon} \longrightarrow \chi^{\alpha}(y) v_{0}(x, y) \quad \text { and } \quad \varepsilon^{\lambda / 2} \widetilde{\nabla v}_{\varepsilon} \longrightarrow 0 \text {. }
$$

Proof (a) If $\Omega_{\varepsilon}^{\alpha}$ is connected, theorem 4.6 in [31] yields the result. If $\Omega_{\varepsilon}^{\alpha}$ is disconnected, however, only the first part of this proof can be carried out leading to the reduced result.

(b) The proof can be obtained by the path described in the proof of proposition 1.14 in [28] (which is stated for $\chi^{\alpha}=1$ in $Y$ ) but using part (b) instead of part (a) of theorem 3.3 .

(d) In any of the previous cases, i.e. (a), (b) and (c), the estimate (27) is satisfied with $\lambda=2$ (since $\varepsilon \leq 1$ ). Thus, theorem 2.1 as well as propositions 2.5 and 2.6 in [29] yield the result.

(c) The estimate (27) with $\lambda<2$ particularly implies (27) with $\lambda=2$. Therefore, the conclusions from part (b) apply and we know from part (d) that the trace of $v_{\varepsilon}$ two-scale converges to the trace of the limit function on $\Gamma$. Moreover, the estimate (27) with $\lambda<2$ is in fact stronger than with $\lambda=2$ and we also have the existence of a limit function $\xi_{0} \in L^{2}(\Omega \times Y)$ such that $\varepsilon^{\lambda / 2} \widetilde{\nabla v}_{\varepsilon}$ two-scale converges to $\chi^{\alpha}(y) \xi_{0}(x, y)$. Therefore, $\varepsilon \widetilde{\nabla v} v_{\varepsilon}$ two-scale converges to zero. Moreover, for any vector-valued smooth test function $\phi(x, y)$, integration by parts gives

$$
\begin{aligned}
\varepsilon \int_{\Omega_{\varepsilon}^{\alpha}} \nabla v_{\varepsilon}(x) \cdot \phi(x, x / \varepsilon) \mathrm{d} x & =-\varepsilon \int_{\Omega_{\varepsilon}^{\alpha}} v_{\varepsilon}(x) \nabla_{x} \cdot \phi(x, x / \varepsilon) \mathrm{d} x \\
& -\int_{\Omega_{\varepsilon}^{\alpha}} v_{\varepsilon}(x) \nabla_{y} \cdot \phi(x, x / \varepsilon) \mathrm{d} x+\varepsilon \int_{\Gamma_{\varepsilon}} v_{\varepsilon}(x) \phi(x, x / \varepsilon) \cdot \nu \mathrm{d} \sigma_{x} .
\end{aligned}
$$

Passing to the limit in each term gives

$$
0=-\int_{\Omega} \int_{Z^{\alpha}} v_{0}(x, y) \nabla_{y} \cdot \phi(x, y) \mathrm{d} y \mathrm{~d} x+\int_{\Omega} \int_{\Gamma} v_{0}(x, y) \phi(x, y) \cdot \nu \mathrm{d} \sigma_{y} \mathrm{~d} x
$$

and, by integration by parts,

$$
0=\int_{\Omega} \int_{Z^{\alpha}} \nabla_{y} v_{0}(x, y) \cdot \phi(x, y) \mathrm{d} y \mathrm{~d} x
$$

for all $\phi$ implying that $v_{0}$ does not depend on $y$. Since $\varepsilon^{\lambda / 2} \widetilde{\nabla v} v_{\varepsilon}(x)$ is bounded, there exists a limit function $\xi_{0}(x, y) \in\left[L^{2}(\Omega \times Y)\right]^{n}$ such that

$$
\lim _{\varepsilon \rightarrow 0} \int_{\Omega} \varepsilon^{\lambda / 2} \widetilde{\nabla} v_{\varepsilon}(x) \cdot \psi(x, x / \varepsilon) \mathrm{d} x=\int_{\Omega} \int_{Y} \xi_{0}(x, y) \cdot \psi(x, y) \mathrm{d} y \mathrm{~d} x
$$

for all $\psi \in\left[C_{0}^{\infty}\left(\Omega ; C_{\#}^{\infty}(Y)\right)\right]^{n}$ equal to zero if $y \in Y \backslash \bar{Z}^{\alpha}$. Choosing $\psi$ such that $\nabla_{y} \cdot \psi=0$, integration by parts on the left-hand side gives

$$
\int_{\Omega} \int_{Y} \xi_{0}(x, y) \cdot \psi(x, y) \mathrm{d} y \mathrm{~d} x=0
$$

for all divergence-free $\psi$. Therefore, $\xi_{0}$ can be expressed as the gradient of a function $v_{1} \in L^{2}\left(\Omega ; W_{\#}^{1,2}\left(Z^{\alpha}\right) / \mathbb{R}\right), \xi_{0}=\nabla_{y} u_{1}$. 
(e) The same considerations as for part (b) can be carried out but with $\xi_{0}$ being the two-scale limit of $\varepsilon^{\lambda / 2} \widetilde{\nabla v_{\varepsilon}}$ (instead of $\varepsilon \widetilde{\nabla v} v_{\varepsilon}$ ). The argument using integration by parts then reads as

$$
\begin{aligned}
\int_{\Omega} \int_{Y} \xi_{0}(x, y) & \psi \psi(x, y) \mathrm{d} y \mathrm{~d} x \\
& =-\lim _{\varepsilon \rightarrow 0} \varepsilon^{\lambda / 2} \int_{\Omega_{\varepsilon}^{\alpha}} v_{\varepsilon}(x)\left(\nabla_{x} \cdot \psi(x, x / \varepsilon)+\varepsilon^{-1} \nabla_{y} \cdot \psi(x, x / \varepsilon)\right) \mathrm{d} x=0
\end{aligned}
$$

for all $\psi$, implying assertion (e).

Now, some deeper results about the convergence of the traces on $\Gamma_{\varepsilon}$ need to be obtained in order to allow the identification of the limit problems. For this purpose, two technical lemmas are cited first, which allow the formulation of a theorem describing the limit behaviour of the sequence of traces on $\Gamma_{\varepsilon}$. This theorem generalises theorem 3.2 of [20], which is stated for $k=l=0$, to the case $0 \leq k=$ $l<2$ and a corollary is added which discusses the behaviour if $k \neq l$. For ease of notation, let the mean value of a function $v \in L^{2}(Y)$ be denoted by $m(v)$, i.e.

$$
m(v)=\int_{Y} v(y) \mathrm{d} y,
$$

and the function mapping $v_{\varepsilon} \in L^{2}\left(\Omega_{\varepsilon}\right)$ to its mean value in each cell be denoted by $m_{\varepsilon}\left(v_{\varepsilon}\right)$ whose (constant) value in each $Y_{\varepsilon}^{k}$ is given by

$$
m_{\varepsilon}^{k}\left(v_{\varepsilon}\right)=\varepsilon^{-n} \int_{\varepsilon Y_{k}} v_{\varepsilon}(y) \mathrm{d} y
$$

where $\Omega_{\varepsilon}=\Omega \cap \operatorname{int} \bigcup_{\alpha} \bar{\Omega}_{\varepsilon}^{\alpha}$. Moreover, the function $m_{\varepsilon}^{*}\left(v_{\varepsilon}\right)=\left(\left(m_{\varepsilon}^{*}\left(v_{\varepsilon}\right)\right)_{p}\right)_{p=1, \ldots, n}$ is, constant in each cell, defined by

$$
\left(m_{\varepsilon}^{*}\left(v_{\varepsilon}\right)\right)_{p}=\varepsilon^{-1}\left[m_{\varepsilon}^{p(k)}\left(v_{\varepsilon}\right)-m_{\varepsilon}^{k}\left(v_{\varepsilon}\right)\right]
$$

in each $Y_{\varepsilon}^{k}$ for all $p \in\{1, \ldots, n\}$, where $p(k)$ denotes the operator changing the $p$ th component of $k,(k)_{p}$, into $(k)_{p}+1$, i.e. $p(k)=k+e_{p}$. This is to be understood such that $m_{\varepsilon}^{p(k)}\left(v_{\varepsilon}\right)=0$ if $Y_{\varepsilon}^{p(k)} \cap \Omega=\emptyset$. Moreover, we write $\partial_{p}=\partial_{e_{p}}$ for ease of notation.

For future reference, we cite the following two lemmas, the proofs of which can be found in [20] (lemmas 3.2 and 3.4 in [20], respectively).

\section{Lemma 3.5}

(a) Let $v$ be a function in $L^{2}(Y)$ such that $v^{\alpha}=\left.v\right|_{Z^{\alpha}} \in W_{\#}^{1,2}\left(Z^{\alpha}\right)$ for $\alpha \in\{\mathrm{p}, \mathrm{s}\}$. Then, there exists a positive constant $C$ (only dependent on $Y$ ) such that

$$
|v-m(v)|_{Y}^{2} \leq C\left[\left|\nabla v^{\mathrm{p}}\right|_{Z^{\mathrm{p}}}^{2}+\left|\nabla v^{\mathrm{s}}\right|_{Z^{\mathrm{s}}}^{2}+\left|v^{\mathrm{p}}-v^{\mathrm{s}}\right|_{\Gamma}^{2}\right]
$$

(b) For a function $v_{\varepsilon}$ in $L^{2}\left(\Omega_{\varepsilon}\right)$ such that $v_{\varepsilon}^{\alpha}=\left.u_{\varepsilon}\right|_{\Omega_{\varepsilon}^{\alpha}} \in W^{1,2}\left(\Omega_{\varepsilon}^{\alpha}\right)$ for $\alpha \in\{\mathrm{p}, \mathrm{s}\}$, there exists a positive constant $C$ (only dependent on $\Omega$ ) such that

$$
\left|v_{\varepsilon}-m_{\varepsilon}\left(v_{\varepsilon}\right)\right|_{\Omega_{\varepsilon}}^{2} \leq C\left[\varepsilon^{2}\left|\nabla v_{\varepsilon}^{\mathrm{p}}\right|_{\Omega_{\varepsilon}^{\mathrm{p}}}^{2}+\varepsilon^{2}\left|\nabla v_{\varepsilon}^{\mathrm{s}}\right|_{\Omega_{\varepsilon}^{\mathrm{s}}}^{2}+\varepsilon\left|v_{\varepsilon}^{\mathrm{p}}-v_{\varepsilon}^{\mathrm{s}}\right|_{\Gamma_{\varepsilon}}^{2}\right] .
$$




\section{Lemma 3.6}

Denote $\Omega_{\varepsilon}^{*}=\Omega \backslash \Gamma_{\varepsilon}, Y^{*}=Y \backslash \Gamma$ and $Y_{\varepsilon}^{* k}=Y_{\varepsilon}^{k} \cap \Omega_{\varepsilon}$ and let $w_{\varepsilon}$ be a sequence in $L^{2}(\Omega)$ satisfying

( $\alpha) w_{\varepsilon} \in W^{1,2}\left(Y_{\varepsilon}^{* k}\right)$ for all $k$;

( $\beta$ ) for all $p \in\{1, \ldots, n\}$ there exists $w_{\varepsilon}^{p}$ such that $\partial_{p} w_{\varepsilon}^{p} \in L^{2}\left(\Omega_{\varepsilon}^{*}\right), \partial_{p} w_{\varepsilon}^{p}=\partial_{p} w_{\varepsilon}$ in every $Y_{\varepsilon}^{* k}$ and $\left.w_{\varepsilon}^{p}\right|_{\Omega_{\varepsilon}^{\mathrm{p}}}-\left.w_{\varepsilon}^{p}\right|_{\Omega_{\varepsilon}^{\mathrm{s}}}=\left.w_{\varepsilon}\right|_{\Omega_{\varepsilon}^{\mathrm{p}}}-\left.w_{\varepsilon}\right|_{\Omega_{\varepsilon}^{\mathrm{s}}}$ on $\Gamma_{\varepsilon}$;

$(\gamma)\left|w_{\varepsilon}\right|_{\Omega}+\sum_{p=1}^{n}\left|w_{\varepsilon}^{p}\right|_{\Omega}+\sum_{k}\left|\varepsilon \nabla w_{\varepsilon}\right|_{Y_{\varepsilon}^{* k}} \leq C$.

Then, there exists a $w \in L^{2}\left(\Omega ; W_{\#}^{1,2}\left(Y^{*}\right)\right)$ such that, up to a subsequence, $w_{\varepsilon}$ twoscale converges to $w$, the sequence $\xi_{\varepsilon}$ defined by $\xi_{\varepsilon}=\varepsilon \nabla w_{\varepsilon}$ in each $Y_{\varepsilon}^{* k}$ two-scale converges to $\nabla_{y} w$ and $\left(\left.w_{\varepsilon}\right|_{\Omega_{\varepsilon}^{\mathrm{p}}}-\left.w_{\varepsilon}\right|_{\Omega_{\varepsilon}^{\mathrm{s}}}\right)$ two-scale converges on $\Gamma_{\varepsilon}$ to $\left(\left.w\right|_{Z^{\mathrm{p}}}-\left.w\right|_{Z^{\mathrm{s}}}\right)$ in the sense (24).

Using the preceding two lemmas, the convergence of a sequence in $L^{2}\left(\Omega_{\varepsilon}\right)$ such that $v_{\varepsilon}^{\alpha}=\left.v\right|_{\Omega_{\varepsilon}^{\alpha}} \in W^{1,2}\left(\Omega_{\varepsilon}^{\alpha}\right)$ for $\alpha \in\{\mathrm{p}, \mathrm{s}\}$ can be obtained where the scaling exponents of the gradients in each part of $\Omega_{\varepsilon}$ are the same.

\section{Proposition 3.7}

Let $0 \leq \lambda<2$ be given and $v_{\varepsilon}$ be a sequence of functions in $L^{2}\left(\Omega_{\varepsilon}\right)$ such that $v_{\varepsilon}^{\alpha}=\left.v\right|_{\Omega_{\varepsilon}^{\alpha}} \in W^{1,2}\left(\Omega_{\varepsilon}^{\alpha}\right)$ for $\alpha \in\{\mathrm{p}, \mathrm{s}\}$ such that

$$
\left|v_{\varepsilon}\right|_{\Omega_{\varepsilon}}+\varepsilon^{\lambda / 2}\left|\nabla v_{\varepsilon}^{\mathrm{p}}\right|_{\Omega_{\varepsilon}^{\mathrm{p}}}+\varepsilon^{\lambda / 2}\left|\nabla v_{\varepsilon}^{\mathrm{s}}\right|_{\Omega_{\varepsilon}^{\mathrm{s}}}+\varepsilon^{(\lambda-1) / 2}\left|v_{\varepsilon}^{\mathrm{p}}-v_{\varepsilon}^{\mathrm{s}}\right|_{\Gamma_{\varepsilon}} \leq C .
$$

Moreover, let the sequence $w_{\varepsilon}$ defined by

$$
w_{\varepsilon}=\varepsilon^{\lambda / 2-1}\left(v_{\varepsilon}-m_{\varepsilon}\left(v_{\varepsilon}\right)\right)-\theta(\lambda) z_{\varepsilon} \cdot m_{\varepsilon}^{*}\left(v_{\varepsilon}\right)
$$

with $z_{\varepsilon}=x / \varepsilon-k+(1 / 2, \ldots, 1 / 2)^{T}$ in $Y_{\varepsilon}^{k}$. Then, the sequences $u_{\varepsilon}$ and $\varepsilon^{\lambda / 2} \widetilde{\nabla v_{\varepsilon}^{\alpha}}$ converge to the limit functions $v_{0}$ and $\theta(\lambda) \nabla v_{0}+\nabla_{y} v_{1}$ given in theorem 3.4 and the following statements hold:

(a) The sequences $w_{\varepsilon}$ and $\xi_{\varepsilon}^{\alpha}$ (as defined in lemma 3.6), up to subsequences, twoscale converge to $v_{1} \in L^{2}(\Omega \times Y)$ with $v_{1}^{\alpha}=\left.u_{1}\right|_{Z^{\alpha}} \in L^{2}\left(\Omega ; W^{1,2}\left(Z^{\alpha}\right) / \mathbb{R}\right)$ and $\nabla_{y} v_{1}^{\alpha}$ for $\alpha \in\{\mathrm{p}, \mathrm{s}\}$, respectively.

(b) The sequence $\left.\varepsilon^{\lambda / 2-1}\left(v_{\varepsilon}^{\mathrm{p}}-v_{\varepsilon}^{\mathrm{s}}\right)\right|_{\Gamma_{\varepsilon}}$ two-scale converges to $\left.\left(v_{1}^{\mathrm{p}}-v_{1}^{\mathrm{s}}\right)\right|_{\Gamma}$ in the sense (24).

Proof The idea of proof is to show that $w_{\varepsilon}$ satisfies the requirements of lemma 3.6 and the limit function of $w_{\varepsilon}$ is actually $v_{1}$. Then, all conclusions directly follow from lemma 3.6, especially part (b) since $\left.\varepsilon^{\lambda / 2-1}\left(v_{\varepsilon}^{\mathrm{p}}-v_{\varepsilon}^{\mathrm{s}}\right)\right|_{\Gamma_{\varepsilon}}=\left.\left(w_{\varepsilon}^{\mathrm{p}}-w_{\varepsilon}^{\mathrm{s}}\right)\right|_{\Gamma_{\varepsilon}}$. In order to apply lemma 3.6, the requirements $(\alpha),(\beta)$ and $(\gamma)$ need to be verified where, in particular, the functions $w_{\varepsilon}^{p}$ need to be chosen as

$$
w_{\varepsilon}^{p}=w_{\varepsilon}+\theta(\lambda) \sum_{q \neq p}\left(z_{\varepsilon}\right)_{q}\left(m_{\varepsilon}^{*}\left(v_{\varepsilon}\right)\right)_{q} .
$$


For $\lambda=0$, the verification of the prerequisites of lemma 3.6 can be found in [20] (cf. the proof of theorem 3.2). Therefore, only the case $0<\lambda<2$ is presented here.

For $\lambda \neq 0$, points $(\alpha)$ and $(\beta)$ of lemma 3.6 are clear. It therefore only remains to be shown that the estimate from point $(\gamma)$ is fulfilled. Applying lemma 3.5 to $v_{\varepsilon}$, we obtain

$$
\left|w_{\varepsilon}\right|_{\Omega_{\varepsilon}}^{2}=\varepsilon^{\lambda-2}\left|v_{\varepsilon}-m_{\varepsilon}\left(v_{\varepsilon}\right)\right|_{\Omega_{\varepsilon}}^{2} \leq C\left[\varepsilon^{\lambda}\left|\nabla v_{\varepsilon}^{\mathrm{p}}\right|_{\Omega_{\varepsilon}^{\mathrm{p}}}^{2}+\varepsilon^{\lambda}\left|\nabla v_{\varepsilon}^{\mathrm{s}}\right|_{\Omega_{\varepsilon}^{\mathrm{s}}}^{2}+\varepsilon^{\lambda-1}\left|v_{\varepsilon}^{\mathrm{p}}-v_{\varepsilon}^{\mathrm{s}}\right|_{\Gamma_{\varepsilon}}^{2}\right] \leq C,
$$

where the last step follows from the prerequisite (37). Since, moreover, $w_{\varepsilon}^{p}=w_{\varepsilon}$ and $\varepsilon \nabla w_{\varepsilon}=\varepsilon^{\lambda / 2} \nabla v_{\varepsilon}$, assumption $(\gamma)$ is satisfied. Noting that $\varepsilon \nabla w_{\varepsilon}=\varepsilon^{\lambda / 2} \nabla v_{\varepsilon}$ two-scale converges to $\nabla_{y} v_{1}$, the proof of part (a) of this theorem is also completed.

\section{Remark 3.8}

Moreover, for $\lambda=0$ it can be shown that, up to a subsequence, $m_{\varepsilon}^{*}\left(v_{\varepsilon}\right)$ converges to $\nabla v$ where $v$ is the limit function of $v_{\varepsilon}$ (lemma 3.3 in [20]). In the proof of proposition 3.7 it again becomes clear why for $\lambda=0, \Omega_{\varepsilon}^{\alpha}$ needs to be connected (also cf. theorem 3.4): In this case, the functions $w_{\varepsilon}^{p}$ are defined in a way that requires the connectivity.

\section{Corollary 3.9}

Let $0 \leq \lambda \leq 2$ and $0 \leq k<l \leq 2$ be given and $v_{\varepsilon}$ be a sequence of functions in $L^{2}\left(\Omega_{\varepsilon}\right)$ such that $v_{\varepsilon}^{\alpha}=\left.v_{\varepsilon}\right|_{\Omega_{\varepsilon}^{\alpha}} \in W^{1,2}\left(\Omega_{\varepsilon}^{\alpha}\right)$ for $\alpha \in\{\mathrm{p}, \mathrm{s}\}$ and

$$
\left|v_{\varepsilon}\right|_{\Omega_{\varepsilon}}+\varepsilon^{k / 2}\left|\nabla v_{\varepsilon}^{\mathrm{p}}\right|_{\Omega_{\varepsilon}^{\mathrm{p}}}+\varepsilon^{l / 2}\left|\nabla v_{\varepsilon}^{\mathrm{s}}\right|_{\Omega_{\varepsilon}^{\mathrm{s}}}+\varepsilon^{(\lambda-1) / 2}\left|v_{\varepsilon}^{\mathrm{p}}-v_{\varepsilon}^{\mathrm{s}}\right|_{\Gamma_{\varepsilon}} \leq C .
$$

If $\lambda=k$, let

$$
\varepsilon^{(k-1) / 2}\left|v_{\varepsilon}^{\mathrm{p}}\right|_{\Gamma_{\varepsilon}}+\varepsilon^{(k-1) / 2}\left|v_{\varepsilon}^{\mathrm{s}}\right|_{\Gamma_{\varepsilon}} \leq C
$$

additionally be satisfied. Then, the sequence $\left.\varepsilon^{\lambda / 2-1}\left(v_{\varepsilon}^{\mathrm{p}}-v_{\varepsilon}^{\mathrm{s}}\right)\right|_{\Gamma_{\varepsilon}}$ two-scale converges in the sense (24) and the limit is given by

(a) $-\left.v_{1}^{\mathrm{s}}\right|_{\Gamma}$, if $\lambda=l$,

(b) $\left.\left(v_{1}^{\mathrm{p}}+\theta(k) y \cdot \nabla v\right)\right|_{\Gamma}$, if $\lambda=k$.

Note that $v_{1}^{\mathrm{s}}=v^{\mathrm{s}}$ if $l=2$.

Proof (a) Let $\lambda=l>0$. It is easily seen in the proof of proposition 3.7 that if the gradients in $\Omega_{\varepsilon}^{\mathrm{p}}$ satisfy a better estimate (i.e. with exponent $k / 2$ in (37)), the sequence $\varepsilon \nabla w_{\varepsilon}=\varepsilon^{l / 2} \nabla v_{\varepsilon}$ in the proof of proposition 3.7 two-scale converges to zero in $Z^{\mathrm{p}}$ and to $\nabla_{y} v_{1}^{\mathrm{s}}$ in $Z^{\mathrm{s}}$. Therefore, it follows from proposition 3.7 that $\left.\varepsilon^{l / 2-1}\left(v_{\varepsilon}^{\mathrm{p}}-v_{\varepsilon}^{\mathrm{s}}\right)\right|_{\Gamma_{\varepsilon}}$ two-scale converges to $-\left.v_{1}^{\mathrm{s}}\right|_{\Gamma}$.

(b) Let $\lambda=k$ and $w_{\varepsilon}$ be defined as in proposition 3.7. The proof is given using a bootstrap argument. In contrast to assumption (40), assume, for now, the weaker estimate

$$
\varepsilon^{(k-1) / 2}\left|v_{\varepsilon}^{\mathrm{p}}\right|_{\Gamma_{\varepsilon}}+\varepsilon^{(l-1) / 2}\left|v_{\varepsilon}^{\mathrm{s}}\right|_{\Gamma_{\varepsilon}} \leq C .
$$


Notice that, in any case, $w_{\varepsilon}$ does not meet the assumptions of lemma 3.6 as the $\varepsilon$-power in front of the term $\left|\nabla v_{\varepsilon}^{\mathrm{s}}\right|_{\Omega_{\varepsilon}^{\mathrm{s}}}$ in the estimate (39) is not sufficient for $(\gamma)$ to hold. Therefore, denote by $\bar{w}_{\varepsilon}$ and $\hat{w}_{\varepsilon}$ the functions defined as follows,

$$
\bar{w}_{\varepsilon}=\left\{\begin{array}{ll}
\varepsilon^{k / 2-1}\left(v_{\varepsilon}^{\mathrm{p}}-m_{\varepsilon}\left(v_{\varepsilon}\right)\right), & x \in \Omega_{\varepsilon}^{\mathrm{p}}, \\
0, & x \in \Omega_{\varepsilon}^{\mathrm{s}},
\end{array} \quad \hat{w}_{\varepsilon}= \begin{cases}0, & x \in \Omega_{\varepsilon}^{\mathrm{p}}, \\
\varepsilon^{l / 2-1}\left(v_{\varepsilon}^{\mathrm{s}}-m_{\varepsilon}\left(v_{\varepsilon}\right)\right), & x \in \Omega_{\varepsilon}^{\mathrm{s}} .\end{cases}\right.
$$

Letting $\bar{w}_{\varepsilon}^{p}=\bar{w}_{\varepsilon}, \bar{w}_{\varepsilon}$ satisfies the requirements of lemma 3.6 and $\varepsilon \nabla \bar{w}_{\varepsilon}$ two-scale converges as follows,

$$
\varepsilon \nabla \bar{w}_{\varepsilon}=\left\{\begin{array} { l l } 
{ \varepsilon ^ { k / 2 } \nabla v _ { \varepsilon } ^ { \mathrm { p } } , } & { x \in \Omega _ { \varepsilon } ^ { \mathrm { p } } , } \\
{ 0 , } & { x \in \Omega _ { \varepsilon } ^ { \mathrm { s } } , }
\end{array} \longrightarrow \left\{\begin{array}{ll}
\nabla_{y} v_{1}^{\mathrm{p}}+\theta(k) \nabla_{x} v^{\mathrm{p}}, & x \in \Omega, y \in Z^{\mathrm{p}}, \\
0, & x \in \Omega, y \in Z^{\mathrm{s}}
\end{array}\right.\right.
$$

Therefore, up to a constant,

$$
\bar{w}_{\varepsilon} \rightarrow \begin{cases}v_{1}^{\mathrm{p}}+\theta(k) y \cdot \nabla_{x} v^{\mathrm{p}}, & x \in \Omega, y \in Z^{\mathrm{p}}, \\ 0, & x \in \Omega, y \in Z^{\mathrm{s}} .\end{cases}
$$

Now, consider $\hat{w}_{\varepsilon}$. Similarly as for $\bar{w}_{\varepsilon}$, it can be shown that

$$
\hat{w}_{\varepsilon} \rightarrow \begin{cases}0, & x \in \Omega, y \in Z^{\mathrm{p}}, \\ v_{1}^{\mathrm{s}}, & x \in \Omega, y \in Z^{\mathrm{s}} .\end{cases}
$$

in two-scale sense. Therefore,

$$
\left.\varepsilon^{k / 2-1}\left(v_{\varepsilon}^{\mathrm{p}}-v_{\varepsilon}^{\mathrm{s}}\right)\right|_{\Gamma_{\varepsilon}}=\left.\left(\bar{w}_{\varepsilon}^{\mathrm{p}}-\varepsilon^{(k-l) / 2} \hat{w}_{\varepsilon}^{\mathrm{s}}\right)\right|_{\Gamma_{\varepsilon}} .
$$

It is known from the a-priori estimate that this term converges, as do $\left.\bar{w}_{\varepsilon}^{\mathrm{p}}\right|_{\Gamma_{\varepsilon}}$ and $\left.\hat{w}_{\varepsilon}^{\mathrm{s}}\right|_{\Gamma_{\varepsilon}}$. Therefore, $\left.\hat{w}_{\varepsilon}^{\mathrm{s}}\right|_{\Gamma_{\varepsilon}}$ has to converge with order $\varepsilon^{(l-k) / 2}$ at least. Since prerequisite (40) gives an even better estimate than what we have assumed so far, the term must converge with order greater than $\varepsilon^{(l-k) / 2}$ and therefore, $\left.\varepsilon^{(k-l) / 2} \bar{w}_{\varepsilon}^{\mathrm{s}}\right|_{\Gamma_{\varepsilon}} \rightarrow 0$ in twoscale sense. Altogether, we have

$$
\left.\left.\varepsilon^{k / 2-1}\left(v_{\varepsilon}^{\mathrm{p}}-v_{\varepsilon}^{\mathrm{s}}\right)\right|_{\Gamma_{\varepsilon}} \rightarrow\left(v_{1}^{\mathrm{p}}+\theta(k) y \cdot \nabla v^{\mathrm{p}}\right)\right|_{\Gamma} .
$$

\section{Remark 3.10}

As discussed in the beginning of this subsection, it does not pose problems if the sequence of functions depends on further variables, as time for example. Theorem 3.4 can be directly adapted to bounded sequences in $L^{2}\left(0, T ; W^{1,2}(\Omega)\right)$. If, moreover, the sequence of time derivatives is bounded in $L^{2}(\Omega \times S)$, the sequence of time derivatives also converges and its limit is the time derivative of the limit of the original sequence. If we only have the boundedness of the time derivatives in $L^{2}\left(0, T ;\left(W^{1,2}(\Omega)\right)^{\prime}\right)$, the limit passage can be carried out after integrating by parts with respect to time. Another integration by parts in the corresponding limit term yields the expected result. For details, we refer to [33], [30] and [34]. 


\section{Determination of the limit problems}

Using the results from the previous section, the sequences of solutions of problem (4) corresponding to a sequence of parameters $\varepsilon$ approaching zero yield two-scale limits. In this section, the limit problems satisfied by the limit of the sequences solutions are identified depending on the choice of parameters. Explicitly, it is shown that each limit actually satisfies the corresponding limit problem stated in $\S 2$. A closer examination of the convergence of the interfacial-exchange term depending on $m$ (cf. lemma 4.1) motivates the separate treatment of the cases $m \geq 1$ and $m<1$.

With the a-priori estimates for the sequences of solutions given by theorem 3.1, the convergence of the functions $u_{\varepsilon}^{\alpha}$ and $\varepsilon^{\lambda} \nabla u_{\varepsilon}^{\alpha}$ follows directly from theorems 3.3, 3.4 and by remark 3.10. From theorem 3.4, propostion 3.7 and corollary 3.9, some more information about the interfacial-exchange term can be obtained. For ease of notation, we write

$$
f_{1}^{\mathrm{ex}}(x, y, t)=a(x, y, t)\left(u_{1}^{\mathrm{p}}(x, y, t)-u_{1}^{\mathrm{s}}(x, y, t)\right)
$$

and we also refer to the definition of $f^{\text {ex }}$ (cf. (19)). If $k \neq l$ and $m \leq k-1$, we assume that (40) is satisfied.

\section{Lemma 4.1}

There exists a limit function $\xi^{\mathrm{ex}} \in L^{2}\left(0, T ; L^{2}(\Omega \times Y)\right)$ such that the scaled interfacialexchange term given by $\varepsilon^{\mu} f_{\varepsilon}^{\mathrm{ex}}(x, t)$ two-scale converges to

$$
\begin{cases}\xi^{\mathrm{ex}}, & \text { if } \mu=(m-1) / 2, \\ 0, & \text { if } \mu>(m-1) / 2,\end{cases}
$$

in two-scale sense (cf. (24)). For some cases, the limit function can be further specified:

(a) If $k, l \leq 2$ and $m=1$, the limit function is given by $\xi^{\mathrm{ex}}=f^{\mathrm{ex}}$.

(b) If $k=l<2$ and $m=k-1$, the limit function is given by $\xi^{\mathrm{ex}}=f_{1}^{\mathrm{ex}}$.

(c) If $k \neq l$, the limit function is given by $\xi^{\mathrm{ex}}=a\left(u_{1}^{\mathrm{p}}+\theta(k) y \cdot \nabla u\right)$ if $m=k-1$ and by $\xi^{\mathrm{ex}}=-a u_{1}^{\mathrm{s}}$ if $m=l-1$.

Moreover, for $m<1, f_{\varepsilon}^{\mathrm{ex}}(x, t)$ converges to zero, implying, if $k, l \leq 2, u^{\mathrm{p}}(x, y, t)=$ $u^{\mathrm{s}}(x, y, t)$ for a.e. $x \in \Omega, y \in \Gamma, t \in S$.

Proof By theorem 3.1 the norm of the difference in the interfacial-exchange term is bounded in the following way,

$$
\varepsilon^{(m-1) / 2} \sqrt{\varepsilon}\left|u_{\varepsilon}^{\mathrm{p}}-u_{\varepsilon}^{\mathrm{s}}\right|_{\Gamma_{\varepsilon}, t} \leq C
$$

for a.e. $t \in S$. Therefore, theorem 3.4 (d) yields the existence of a limit function $\xi^{\mathrm{ex}} \in L^{2}\left(0, T ; L^{2}(\Omega \times Y)\right)$ such that

$$
\begin{aligned}
\lim _{\varepsilon \rightarrow 0} \varepsilon \int_{S} \int_{\Gamma_{\varepsilon}} \varepsilon^{(m-1) / 2} a(x, x / \varepsilon, t) & \left(u_{\varepsilon}^{\mathrm{p}}(x, t)-u_{\varepsilon}^{\mathrm{s}}(x, t)\right) \phi(x, x / \varepsilon, t) \mathrm{d} \sigma_{x} \mathrm{~d} t \\
& =\int_{S} \int_{\Omega \times \Gamma} a(x, y, t) \xi^{\mathrm{ex}}(x, y, t) \phi(x, y, t) \mathrm{d} \sigma_{y} \mathrm{~d} x \mathrm{~d} t
\end{aligned}
$$


for all smooth $\phi$. This implies that for $\mu=(m-1) / 2, \varepsilon^{\mu} f_{\varepsilon}^{\text {ex }}$ two-scale converges (in the sense (24)) to some limit function $\xi^{\text {ex }}$, which, by theorem 3.4, is given by $\xi^{\mathrm{ex}}=a\left(u^{\mathrm{p}}-u^{\mathrm{s}}\right)$ if $k, l \leq 2$ and $m=1$. For $\mu>(m-1) / 2$, it is clear that $\varepsilon^{\mu} f_{\varepsilon}^{\mathrm{ex}}$ two-scale converges to zero. Assertions (b) and (c), follow from proposition 3.7 and corollary 3.9.

For $m<1$, it especially follows from $(\dagger)$

$$
\varepsilon\left|u_{\varepsilon}^{\mathrm{p}}-u_{\varepsilon}^{\mathrm{s}}\right|_{\Gamma_{\varepsilon}, T}^{2} \leq \varepsilon^{1-m} C \leq C
$$

(since $\varepsilon \leq 1$ ), which, by theorem $3.4(\mathrm{~d})$, yields

$$
\left|\xi^{\mathrm{ex}}\right|_{\Omega \times \Gamma, T}^{2} \leq \lim _{\varepsilon \rightarrow 0} \varepsilon\left|u_{\varepsilon}^{\mathrm{p}}-u_{\varepsilon}^{\mathrm{s}}\right|_{\Gamma_{\varepsilon}, T}^{2} \leq \lim _{\varepsilon \rightarrow 0} \varepsilon^{1-m} C .
$$

Passing to the limit yields the second assertion for the limit of the interfacialexchange term.

\section{Remark 4.2}

Note that from theorem 3.4 and lemma 4.1 it directly follows that if $m<1$ and one of the diffusion scaling exponents is smaller than 2 , i.e. $0 \leq k<2$ or $0 \leq l<2$, both limit functions are constant with respect to the microvariable $y$ on $\Gamma$.

\subsection{The case $m \geq 1$}

If $m \geq 1$, two equations appear in the macroscopic limit. It is therefore sufficient to investigate the limit problem of one of the unknowns, of $u^{\alpha}$, say. Since the traces on $\Gamma_{\varepsilon}$ need to converge in order to be able to relate the limit of the interfacial-exchange term to the limit of the solutions, $m>1$ is required if $l>2$ (cf. theorem 3.4).

\section{Proposition 4.3}

Let $m \geq 1$. For $\lambda=0$ and $\Omega_{\varepsilon}^{\alpha}$ connected, the limit function $u^{\alpha}$ associated with the sequence of solutions $u_{\varepsilon}^{\alpha}$ satisfies the weak macromodel equation (20).

PROOF Integration of the weak micromodel equation (4) for $u_{\varepsilon}^{\alpha}$ with respect to the time variable and choosing the test function to be of the form

$$
\phi(x, y, t)=\phi_{0}(x, t)+\varepsilon \phi_{1}(x, x / \varepsilon, t)
$$

with $\left(\phi_{0}, \phi_{1}\right) \in C_{0}^{\infty}\left(S ; C^{\infty}(\Omega)\right) \times C_{0}^{\infty}\left(S ; C^{\infty}\left(\Omega ; C_{\#}^{\infty}(Y)\right)\right)$ gives

$$
\begin{aligned}
0= & \int_{S \times \Omega} \partial_{t} u_{\varepsilon}^{\alpha}(x, t) \chi^{\alpha}(x / \varepsilon)\left[\phi_{0}(x, t)+\varepsilon \phi_{1}(x, x / \varepsilon, t)\right] \mathrm{d} x \mathrm{~d} t \\
& +\int_{S \times \Omega} D^{\alpha}(x, x / \varepsilon, t) \nabla_{x} u_{\varepsilon}^{\alpha}(x, t) \chi^{\alpha}(x / \varepsilon) \\
& \times\left[\nabla_{x} \phi_{0}(x, t)+\varepsilon \nabla_{x} \phi_{1}(x, x / \varepsilon, t)+\nabla_{y} \phi_{1}(x, x / \varepsilon, t)\right] \mathrm{d} x \mathrm{~d} t \\
& -\int_{S \times \Omega} f_{\varepsilon}^{\alpha}(x, t) \chi^{\alpha}(x / \varepsilon)\left[\phi_{0}(x, t)+\varepsilon \phi_{1}(x, x / \varepsilon, t)\right] \mathrm{d} x \mathrm{~d} t \\
& -\sigma^{\alpha}(1) \varepsilon \int_{S \times \Gamma_{\varepsilon}} \varepsilon^{m-1} f_{\varepsilon}^{\operatorname{ex}}(x, t)\left[\phi_{0}(y, t)+\varepsilon \phi_{1}(x, x / \varepsilon, t)\right] \mathrm{d} \sigma_{x} \mathrm{~d} t .
\end{aligned}
$$


Note that the test function is from a slightly larger space than the spaces used in $\S 3$. The arguments for the admissibility of these types of test functions can be found in [28] or [32]. The limit as $\varepsilon$ tends to zero is now determined. The functions $u^{\alpha}$ and $u_{1}^{\alpha}$ appearing in this proof are always understood as the limit functions provided by theorem 3.4.

The limits of the four terms in $(\dagger)$ can be computed individually. For the first term, remark 3.10 is applicable while we make use of (5) for the limit passage in the second term (the fact that the limit of the product is equal to the product of the limits follows from theorem 1.8 in [28]). For the third term, the assumption on $f^{\alpha}$ is used (cf. the beginning of $\S 3$ ). The fourth part can be treated using the results from lemma 4.1. Putting everything together,

$$
\begin{aligned}
0= & \left|Z^{\alpha}\right| \int_{S \times \Omega} \partial_{t} u^{\alpha}(x, t) \phi_{0}(x, t) \mathrm{d} x \mathrm{~d} t \\
& +\int_{S \times \Omega} \int_{Z^{\alpha}} D^{\alpha}(x, y, t)\left(\nabla_{x} u^{\alpha}(x, t)+\nabla_{y} u_{1}^{\alpha}(x, y, t)\right) \\
& \times\left[\nabla_{x} \phi_{0}(x, t)+\nabla_{y} \phi_{1}(x, y, t)\right] \mathrm{d} y \mathrm{~d} x \mathrm{~d} t \\
& -\int_{S \times \Omega} \int_{Z^{\alpha}} f^{\alpha}(x, y, t) \mathrm{d} y \phi_{0}(x, t) \mathrm{d} x \mathrm{~d} t \\
& -\sigma^{\alpha}(m) \int_{S \times \Omega} \int_{\Gamma} f^{\text {ex }}(x, y, t) \mathrm{d} \sigma_{y} \phi_{0}(x, t) \mathrm{d} x \mathrm{~d} t
\end{aligned}
$$

for all $\left(\phi_{0}, \phi_{1}\right)$ is obtained. Choosing $\phi_{0} \equiv 0$ yields

$$
\int_{S \times \Omega} \int_{Z^{\alpha}} D^{\alpha}(x, y, t)\left(\nabla_{x} u^{\alpha}(x, t)+\nabla_{y} u_{1}^{\alpha}(x, y, t)\right) \nabla_{y} \phi_{1}(x, y, t) \mathrm{d} y \mathrm{~d} x \mathrm{~d} t=0
$$

for all $\phi_{1} \in C_{0}^{\infty}\left(S ; C^{\infty}\left(\Omega ; C_{\#}^{\infty}(Y)\right)\right)$. Assuming $u_{1}^{\alpha}=\sum_{j=1}^{n} \partial_{x_{j}} u^{\alpha}(x, t) \varsigma_{j}^{\alpha}(x, y, t)$, the equation is satisfied if $\varsigma_{j}^{\alpha}$ is the solution of the cell problem (17) because

$$
\begin{aligned}
0= & \int_{S \times \Omega} \int_{Z^{\alpha}} D^{\alpha}(x, y, t) \\
& \times\left[\sum_{j=1}^{n} \partial_{x_{j}} u^{\alpha}(x, t) \nabla_{y} \varsigma_{j}^{\alpha}(x, y, t) \nabla_{y} \phi_{1}(x, y, t)+\nabla_{x} u^{\alpha}(x, t) \nabla_{y} \phi_{1}(x, y, t)\right] \mathrm{d} y \mathrm{~d} x \mathrm{~d} t \\
= & \int_{S \times \Omega} \int_{Z^{\alpha}} D^{\alpha}(x, y, t) \\
& \times\left[-\sum_{j=1}^{n} \partial_{x_{j}} u^{\alpha}(x, t) e_{j} \nabla_{y} \phi_{1}(x, y, t)+\nabla_{x} u^{\alpha}(x, t) \nabla_{y} \phi_{1}(x, y, t)\right] \mathrm{d} y \mathrm{~d} x \mathrm{~d} t,
\end{aligned}
$$

which is obviously true for all $\phi_{1}$. On the other hand, if $\varsigma_{j}^{\alpha}$ is the solution of the cell problem (17), the equation is satisfied if $u_{1}^{\alpha}=\sum_{j=1}^{n} \partial_{x_{j}} u^{\alpha}(x, t) \varsigma_{j}^{\alpha}(x, y, t)$. 
Choosing $\phi_{1} \equiv 0$ gives

$$
\begin{aligned}
0= & \left|Z^{\alpha}\right| \int_{S \times \Omega} \partial_{t} u^{\alpha}(x, t) \phi_{0}(x, t) \mathrm{d} x \mathrm{~d} t \\
& +\int_{S \times \Omega} \int_{Z^{\alpha}} D^{\alpha}(x, y, t)\left(\nabla_{x} u^{\alpha}(x, t)+\nabla_{y} u_{1}^{\alpha}(x, y, t)\right) \nabla_{x} \phi_{0}(x, t) \mathrm{d} y \mathrm{~d} x \mathrm{~d} t \\
& -\int_{S \times \Omega} \int_{Z^{\alpha}} f^{\alpha}(x, y, t) \mathrm{d} y \phi_{0}(x, t) \mathrm{d} x \mathrm{~d} t \\
& -\sigma^{\alpha}(m) \int_{S \times \Omega} \int_{\Gamma} f^{\operatorname{ex}}(x, y, t) \mathrm{d} \sigma_{y} \phi_{0}(x, t) \mathrm{d} x \mathrm{~d} t
\end{aligned}
$$

for all $\phi_{0} \in C_{0}^{\infty}\left(S ; C^{\infty}(\Omega)\right)$. Using $u_{1}^{\alpha}=\sum_{j=1}^{n} \partial_{x_{j}} u^{\alpha}(x, t) \varsigma_{j}^{\alpha}(x, y, t)$, the second term can be rewritten,

$$
\begin{aligned}
& \int_{S \times \Omega} \int_{Z^{\alpha}} D^{\alpha}(x, y, t)\left(\nabla_{x} u^{\alpha}(x, t)+\nabla_{y} u_{1}^{\alpha}(x, y, t)\right) \nabla_{x} \phi_{0}(x, t) \mathrm{d} y \mathrm{~d} x \mathrm{~d} t \\
& \quad=\int_{S \times \Omega} \sum_{j, k=1}^{n} \int_{Z^{\alpha}} D^{\alpha}(x, y, t)\left(\delta_{j k}+\partial_{y_{k}} \varsigma_{j}^{\alpha}(x, y, t)\right) \mathrm{d} y \partial_{x_{j}} u^{\alpha}(x, t) \partial_{x_{k}} \phi_{0}(x, t) \mathrm{d} x \mathrm{~d} t \\
& \quad=\int_{S \times \Omega} P^{\alpha}(x, t) \nabla_{x} u^{\alpha}(x, t) \nabla_{x} \phi_{0}(x, t) \mathrm{d} x \mathrm{~d} t,
\end{aligned}
$$

where the tensor $P^{\alpha}$ is defined in (18). Thus,

$$
\begin{aligned}
& \left|Z^{\alpha}\right| \int_{S \times \Omega} \partial_{t} u^{\alpha}(x, t) \phi_{0}(x, t) \mathrm{d} x \mathrm{~d} t+\int_{S \times \Omega} P^{\alpha}(x, t) \nabla_{x} u^{\alpha}(x, t) \nabla_{x} \phi_{0}(x, t) \mathrm{d} x \mathrm{~d} t \\
= & \int_{S \times \Omega} \int_{Z^{\alpha}} f^{\alpha}(x, y, t) \mathrm{d} y \phi_{0}(x, t) \mathrm{d} x \mathrm{~d} t+\sigma^{\alpha}(m) \int_{S \times \Omega} \int_{\Gamma} f^{\operatorname{ex}}(x, y, t) \mathrm{d} \sigma_{y} \phi_{0}(x, t) \mathrm{d} x \mathrm{~d} t
\end{aligned}
$$

for all $\phi_{0} \in C_{0}^{\infty}\left(S ; C^{\infty}(\Omega)\right)$.

\section{Proposition 4.4}

Let $\lambda>0$ and $m \geq 1$. The limit function $u^{\alpha}$ associated with the sequence of solutions $u_{\varepsilon}^{\alpha}$ satisfies the weak macromodel equation (20).

Proof The proof can be obtained analogously to that of propostion 4.3. For $\lambda<2$, the test function needs to be chosen as

$$
\phi(x, t)=\phi_{0}(x, t)+\varepsilon^{1-\lambda / 2} \phi_{1}(x, x / \varepsilon, t)
$$

with $\left(\phi_{0}, \phi_{1}\right) \in C_{0}^{\infty}\left(S ; C^{\infty}(\Omega)\right) \times C_{0}^{\infty}\left(S ; C^{\infty}\left(\Omega ; C_{\#}^{\infty}(Y)\right)\right)$ while, for $\lambda \geq 2, \phi(x, t)=$ $\phi_{1}(x, x / \varepsilon, t)$ is the appropriate test function.

\subsection{The case $m<1$}

The case $m<1$ is now considered. Note that in this case, if one of the diffusion scaling exponents is smaller than two, both limit functions are constant with respect 
to the microvariable $y$ on $\Gamma$ (cf. remark 4.2). A general consideration is made first which is then specified depending on the scaling exponents $k, l$ and $m$.

Consider again the space $\mathcal{V}^{*}=\left\{u_{\varepsilon} \in L^{2}\left(0, T ; L^{2}(\Omega)\right)\left|u_{\varepsilon}\right|_{\Omega_{\varepsilon}^{\alpha}} \in \mathcal{V}\left(\Omega_{\varepsilon}^{\alpha}\right)\right\}$. Addition of equations (4a) and (4b) gives

$$
\begin{aligned}
& \left(\partial_{t} u_{\varepsilon}^{\mathrm{p}}(t) \mid \phi(t)\right)_{\Omega_{\varepsilon}^{\mathrm{p}}}+\left(\partial_{t} u_{\varepsilon}^{\mathrm{s}}(t) \mid \phi(t)\right)_{\Omega_{\varepsilon}^{\mathrm{s}}}+\varepsilon^{k}\left(D_{\varepsilon}^{\mathrm{p}}(t) \nabla u_{\varepsilon}^{\mathrm{p}}(t) \mid \nabla \phi(t)\right)_{\Omega_{\varepsilon}^{\mathrm{p}}} \\
& +\varepsilon^{l}\left(D_{\varepsilon}^{\mathrm{s}}(t) \nabla u_{\varepsilon}^{\mathrm{s}}(t) \mid \nabla \phi(t)\right)_{\Omega_{\varepsilon}^{\mathrm{s}}}+\varepsilon^{m}\left(f_{\varepsilon}^{\mathrm{ex}}(t)|\phi|_{\Omega_{\varepsilon}^{\mathrm{p}}}(t)-\left.\phi\right|_{\Omega_{\varepsilon}^{\mathrm{s}}}(t)\right)_{\Gamma_{\varepsilon}} \\
& =\left(f_{\varepsilon}^{\mathrm{p}}(t) \mid \phi(t)\right)_{\Omega_{\varepsilon}^{\mathrm{p}}}+\left(f_{\varepsilon}^{\mathrm{s}}(t) \mid \phi(t)\right)_{\Omega_{\varepsilon}^{\mathrm{s}}}
\end{aligned}
$$

for all $\phi \in \mathcal{V}^{*}$ a.e. in $(0, T)$. Choosing the test function as

$$
\phi(x, t)= \begin{cases}\Theta(k)\left(\phi^{0}(x, t)+\varepsilon^{1-k / 2} \phi^{\mathrm{p}}(x, x / \varepsilon, t)\right)+(1-\Theta(k)) \phi^{\mathrm{p}}(x, x / \varepsilon, t), & x \in \Omega_{\varepsilon}^{\mathrm{p}}, \\ \Theta(l)\left(\phi^{0}(x, t)+\varepsilon^{1-l / 2} \phi^{\mathrm{s}}(x, x / \varepsilon, t)\right)+(1-\Theta(l)) \phi^{\mathrm{s}}(x, x / \varepsilon, t), & x \in \Omega_{\varepsilon}^{\mathrm{s}},\end{cases}
$$

with $\phi^{0} \in C_{0}^{\infty}\left(S ; C^{\infty}(\Omega)\right), \phi^{\alpha} \in C_{0}^{\infty}\left(S ; C^{\infty}\left(\Omega ; C_{\#}^{\infty}\left(Z^{\alpha}\right)\right)\right)$ and

$$
\Theta(\lambda)= \begin{cases}1, & 0 \leq \lambda<2 \\ 0, & \lambda \geq 2\end{cases}
$$

gives

$$
\begin{aligned}
& \int_{S \times \Omega_{\varepsilon}^{\mathrm{p}}} \partial_{t} u_{\varepsilon}^{\mathrm{p}}(x, t)\left[\Theta(k) \phi^{0}(x, t)+\varepsilon^{1-k / 2} \phi^{\mathrm{p}}(x, x / \varepsilon, t)\right] \mathrm{d} x \mathrm{~d} t \\
& +\int_{S \times \Omega_{\varepsilon}^{\mathrm{s}}} \partial_{t} u_{\varepsilon}^{\mathrm{s}}(x, t)\left[\Theta(l) \phi^{0}(x, t)+\varepsilon^{1-l / 2} \phi^{\mathrm{s}}(x, x / \varepsilon, t)\right] \mathrm{d} x \mathrm{~d} t \\
& +\int_{S \times \Omega_{\varepsilon}^{\mathrm{p}}} D^{\mathrm{p}}(x, x / \varepsilon, t) \varepsilon^{k / 2} \nabla u_{\varepsilon}^{\mathrm{p}}(x, t) \\
& \quad \times\left[\varepsilon^{k / 2} \Theta(k) \nabla_{x} \phi^{0}(x, t)+\varepsilon \nabla_{x} \phi^{\mathrm{p}}(x, x / \varepsilon, t)+\nabla_{y} \phi^{\mathrm{p}}(x, x / \varepsilon, t)\right] \mathrm{d} x \mathrm{~d} t \\
& +\int_{S \times \Omega_{\varepsilon}^{\mathrm{s}}} D^{\mathrm{s}}(x, x / \varepsilon, t) \varepsilon^{l / 2} \nabla u_{\varepsilon}^{\mathrm{s}}(x, t) \\
& +\varepsilon \int_{S \times \Gamma_{\varepsilon}} \varepsilon^{m-1} f_{\varepsilon}^{\mathrm{ex}}(x, t)(\Theta(k)-\Theta(l)) \phi^{0}(x, t) \mathrm{d} \sigma_{x} \mathrm{~d} t \\
& +\varepsilon \int_{S \times \Gamma_{\varepsilon}} f_{\varepsilon}^{\mathrm{ex}}(x, t)\left[\varepsilon^{m-k / 2} \phi^{\mathrm{p}}(x, x / \varepsilon, t)-\varepsilon^{m-l / 2} \phi^{\mathrm{s}}(x, x / \varepsilon, t)\right] \mathrm{d} \sigma_{x} \mathrm{~d} t \\
& -\int_{S \times \Omega_{\varepsilon}^{\mathrm{p}}} f_{\varepsilon}^{\mathrm{p}}(x, t)\left[\Theta(k) \phi^{0}(x, t)+\varepsilon^{1-k / 2} \phi^{\mathrm{p}}(x, x / \varepsilon, t)\right] \mathrm{d} x \mathrm{~d} t \\
& -\int_{S \times \Omega_{\varepsilon}^{\mathrm{s}}} f_{\varepsilon}^{\mathrm{s}}(x, t)\left[\Theta(l) \phi^{0}(x, t)+\varepsilon^{1-l / 2} \phi^{\mathrm{s}}(x, x / \varepsilon, t)\right] \mathrm{d} x \mathrm{~d} t=0
\end{aligned}
$$

for all $\phi^{0}, \phi^{\mathrm{p}}$ and $\phi^{\mathrm{s}}$ if $k, l \leq 2$ (Otherwise, a simpler system is obtained which is contained implicitly in the subsequent proofs). The limit of each term can now be taken separately making use of the results from $\S 3$. 
The only terms requiring some special attention are the interfacial-exchange terms. We first consider the second interfacial exchange term in (46).

If $k=l$, the interfacial-exchange term reads

$$
\varepsilon \int_{S \times \Gamma_{\varepsilon}} \varepsilon^{m-k / 2} f_{\varepsilon}^{\mathrm{ex}}(x, t)\left[\phi^{\mathrm{p}}(x, x / \varepsilon, t)-\phi^{\mathrm{s}}(x, x / \varepsilon, t)\right] \mathrm{d} \sigma_{x} \mathrm{~d} t .
$$

From lemma 4.1 it follows that this term converges to zero if $m-k / 2>(m-1) / 2$, i.e. $m>k-1$, and to

$$
\int_{S \times \Omega} \int_{\Gamma} f_{1}^{\mathrm{ex}}(x, y, t)\left[\phi^{\mathrm{p}}(x, y, t)-\phi^{\mathrm{s}}(x, y, t)\right] \mathrm{d} \sigma_{y} \mathrm{~d} x \mathrm{~d} t
$$

if $m=k-1$. For $m<k-1$, the term does not converge and therefore, $\phi^{\mathrm{p}}=\phi^{\mathrm{s}}$ on $\Gamma$ needs to be chosen.

If $k \neq l$, the interfacial-exchange term can be written as

$\varepsilon \int_{S \times \Gamma_{\varepsilon}} \varepsilon^{m-k / 2} f_{\varepsilon}^{\text {ex }}(x, t) \phi^{\mathrm{p}}(x, x / \varepsilon, t) \mathrm{d} \sigma_{x} \mathrm{~d} t-\varepsilon \int_{S \times \Gamma_{\varepsilon}} \varepsilon^{m-l / 2} f_{\varepsilon}^{\text {ex }}(x, t) \phi^{\mathrm{s}}(x, x / \varepsilon, t) \mathrm{d} \sigma_{x} \mathrm{~d} t$.

The same arguments as above yield that each term goes to zero if $m>\lambda-1$ and to

$$
-\sigma^{\alpha}(\lambda-m) \int_{S \times \Omega} \int_{\Gamma} \xi^{\mathrm{ex}}(x, y, t) \phi^{\alpha}(x, y, t) \mathrm{d} \sigma_{y} \mathrm{~d} x \mathrm{~d} t
$$

if $m=\lambda-1$. If $\lambda=l$, $\xi^{\mathrm{ex}}=a u_{1}^{\mathrm{s}}$ while if $\lambda=k$, $\xi^{\mathrm{ex}}=a\left(u_{1}^{\mathrm{p}}+\theta(k) y \cdot \nabla u\right)$. For $m<\lambda-1$, the respective term does not converge and therefore, $\phi^{\alpha}=0$ on $\Gamma$ needs to be chosen.

Moreover, if $k<2$ and $l \geq 2$, we also have the term

$$
\varepsilon \int_{S \times \Gamma_{\varepsilon}} \varepsilon^{m-1} f_{\varepsilon}^{\text {ex }}(x, t) \phi^{0}(x, t) \mathrm{d} \sigma_{x} \mathrm{~d} t .
$$

The limit of this term cannot be taken straightforwardly since the estimate on the interfacial-exchange term is not sufficient. Nevertheless, we can apply the boundary condition to obtain

$$
\varepsilon \int_{S \times \Gamma_{\varepsilon}} \varepsilon^{l-1} D_{\varepsilon}^{\mathrm{s}}(x, t) \nabla u_{\varepsilon}^{\mathrm{s}}(x, t) \cdot \nu_{\varepsilon}^{\mathrm{s}} \phi^{0}(x, t) \mathrm{d} \sigma_{x} \mathrm{~d} t .
$$

For $l=2$, assuming that $\varepsilon D_{\varepsilon}^{\mathrm{s}} \nabla u_{\varepsilon}^{\mathrm{s}}(x, t) \cdot \nu_{\varepsilon}^{\mathrm{s}}$ two-scale converges to $D^{\mathrm{s}} \nabla_{y} u^{\mathrm{s}}(x, y, t) \cdot \nu^{\mathrm{s}}$, the limit of this term is given by

$$
\int_{S \times \Omega} \int_{\Gamma} D^{\mathrm{s}}(x, y, t) \nabla_{y} u^{\mathrm{s}}(x, y, t) \cdot \nu^{\mathrm{s}} \mathrm{d} \sigma_{y} \phi^{0}(x, t) \mathrm{d} x \mathrm{~d} t .
$$

For this, it suffices if we have an estimate of the form

$$
\left|\varepsilon^{l-1} \nabla u_{\varepsilon}^{\mathrm{s}}\right|_{\Omega_{\varepsilon}^{\mathrm{s}}, t}^{2}+\varepsilon^{2}\left|\varepsilon^{l-1} \Delta u_{\varepsilon}^{\mathrm{s}}\right|_{\Omega_{\varepsilon}^{\mathrm{s}}, t}^{2} \leq C .
$$

Noting that $l=2$ here, such an estimate is obtainable if the right-hand side, i.e. $f_{\varepsilon}^{\mathrm{s}}$, is sufficiently regular.

Having in mind the above results, the limit problems can be determined. We also refer to definitions (7) and (8). 


\section{Proposition 4.5}

Let $m<1$ and $k=0$. Moreover, let $\Omega_{\varepsilon}^{\mathrm{s}}$ be connected if $l=0$. Then, the respective macroscopic models from $\S 2$ are the limit problems of problem (4).

Proof We only consider the case $k=0$, the proof for $k>0$ can be obtained by the same techniques (see [2]). Since $m<1$, lemma 4.1 gives that the limit functions are equal a.e. on $\Omega \times \Gamma$. Moreover, it is a-priori known that $u^{\mathrm{p}}$ does not depend on $y$ and if $l<2$, neither does $u^{\mathrm{s}}$. Now, three cases need to be distinguished. Either $l=0,0<l<2$ or $l \geq 2$.

In the first case, i.e. $l=0$, the limit problem simplifies to

$$
\begin{aligned}
& \int_{S \times \Omega} \partial_{t} u(x, t) \phi^{0}(x, t) \mathrm{d} x \mathrm{~d} t \\
& +\int_{S \times \Omega} \int_{Z^{\mathrm{p}}} D^{\mathrm{p}}(x, y, t)\left[\nabla_{x} u(x, t)+\nabla_{y} u_{1}^{\mathrm{p}}(x, y, t)\right]\left[\nabla_{x} \phi^{0}(x, t)+\nabla_{y} \phi^{\mathrm{p}}(x, y, t)\right] \mathrm{d} x \mathrm{~d} t \\
& +\int_{S \times \Omega} \int_{Z^{\mathrm{s}}} D^{\mathrm{s}}(x, y, t)\left[\nabla_{x} u(x, t)+\nabla_{y} u_{1}^{\mathrm{s}}(x, y, t)\right]\left[\nabla_{x} \phi^{0}(x, t)+\nabla_{y} \phi^{\mathrm{s}}(x, y, t)\right] \mathrm{d} x \mathrm{~d} t \\
& +\lim _{\varepsilon \rightarrow 0} \varepsilon \int_{S \times \Gamma_{\varepsilon}} \varepsilon^{m} f_{\varepsilon}^{\mathrm{ex}}(x, t)\left[\phi^{\mathrm{p}}(x, x / \varepsilon, t)-\phi^{\mathrm{s}}(x, x / \varepsilon, t)\right] \mathrm{d} \sigma_{x} \mathrm{~d} t \\
& =\int_{S \times \Omega} \int_{Y} f(x, y, t) \mathrm{d} y \phi^{0}(x, t) \mathrm{d} x \mathrm{~d} t .
\end{aligned}
$$

The limit of the interfacial-exchange term is zero if $m>-1$,

$$
\int_{S \times \Omega} \int_{\Gamma} f_{1}^{\mathrm{ex}}(x, y, t)\left[\phi^{\mathrm{p}}(x, y, t)-\phi^{\mathrm{s}}(x, y, t)\right] \mathrm{d} \sigma_{y} \mathrm{~d} x \mathrm{~d} t
$$

if $m=-1$, and $\phi^{\mathrm{p}}=\phi^{\mathrm{s}}$ on $\Gamma$ needs to be demanded if $m<-1$. The usual arguments give that the equation with $\phi^{0}=0$ is satisfied if $u_{1}=\sum_{j=1}^{n} \partial_{x_{j}} u(x, t) \varsigma_{j}^{m, 0}(x, y, t)$, where $\varsigma_{j}^{m, 0}$ is the solution of the cell problem (9) (note that if $m<-1$, the space of test functions in the cell problem needs to be restricted to functions satisfying $\phi^{\mathrm{p}}=\phi^{\mathrm{s}}$ on $\Gamma$ ). Therefore, the limit problem is given by

$$
\begin{aligned}
\int_{S \times \Omega} \partial_{t} u(x, t) \phi^{0}(x, t) \mathrm{d} x \mathrm{~d} t+\int_{S \times \Omega} P^{m, 0}(x, t) & \nabla_{x} u(x, t) \nabla_{x} \phi^{0}(x, t) \mathrm{d} x \mathrm{~d} t \\
& =\int_{S \times \Omega} \int_{Y} f(x, y, t) \mathrm{d} y \phi^{0}(x, t) \mathrm{d} x \mathrm{~d} t
\end{aligned}
$$

for all smooth $\phi^{0}$ where the tensor $P^{m, 0}$ is given by (12).

In the case $0<l<2$, the limit problem simplifies to

$$
\begin{aligned}
& \int_{S \times \Omega} \int_{Y} \partial_{t} u(x, t) \mathrm{d} y \phi^{0}(x, t) \mathrm{d} x \mathrm{~d} t \\
& +\int_{S \times \Omega} \int_{Z^{\mathrm{p}}} D^{\mathrm{p}}(x, y, t)\left[\nabla_{x} u^{\mathrm{p}}(x, t)+\nabla_{y} u_{1}^{\mathrm{p}}(x, y, t)\right]\left[\nabla_{x} \phi^{0}(x, t)+\nabla_{y} \phi^{\mathrm{p}}(x, y, t)\right] \mathrm{d} x \mathrm{~d} t \\
& +\int_{S \times \Omega} \int_{Z^{\mathrm{s}}} D^{\mathrm{s}}(x, y, t) \nabla_{y} u_{1}^{\mathrm{s}}(x, y, t) \nabla_{y} \phi^{\mathrm{s}}(x, y, t) \mathrm{d} x \mathrm{~d} t
\end{aligned}
$$




$$
\begin{aligned}
& +\lim _{\varepsilon \rightarrow 0} \varepsilon \int_{S \times \Gamma_{\varepsilon}} f_{\varepsilon}^{\mathrm{ex}}(x, t)\left[\varepsilon^{m} \phi^{\mathrm{p}}(x, x / \varepsilon, t)-\varepsilon^{m-l / 2} \phi^{\mathrm{s}}(x, x / \varepsilon, t)\right] \mathrm{d} \sigma_{x} \mathrm{~d} t \\
& =\int_{S \times \Omega} \int_{Y} f(x, y, t) \mathrm{d} y \phi^{0}(x, t) \mathrm{d} x \mathrm{~d} t .
\end{aligned}
$$

If $m>l-1(>k-1=-1)$, the limit of the interfacial-exchange term is zero. If $m=l-1(>k-1=-1)$, the limit of the interfacial-exchange term is given by

$$
-\sigma^{\mathrm{s}}(l-m) \int_{S \times \Omega} \int_{\Gamma} a(x, y, t) u_{1}^{\mathrm{s}}(x, y, t) \phi^{\mathrm{s}}(x, y, t) \mathrm{d} \sigma_{y} \mathrm{~d} x \mathrm{~d} t .
$$

Choosing $\phi^{0}=\phi^{\mathrm{p}}=0$,

$$
\begin{aligned}
\int_{S \times \Omega} \int_{Z^{\mathrm{s}}} D^{\mathrm{s}}(x, y, t) \nabla_{y} & u_{1}^{\mathrm{s}}(x, y, t) \nabla_{y} \phi^{\mathrm{s}}(x, y, t) \mathrm{d} x \mathrm{~d} t \\
& =\sigma^{\mathrm{s}}(l-m) \int_{S \times \Omega} \int_{\Gamma} a(x, y, t) u_{1}^{\mathrm{s}}(x, y, t) \phi^{\mathrm{s}}(x, y, t) \mathrm{d} \sigma_{y} \mathrm{~d} x \mathrm{~d} t
\end{aligned}
$$

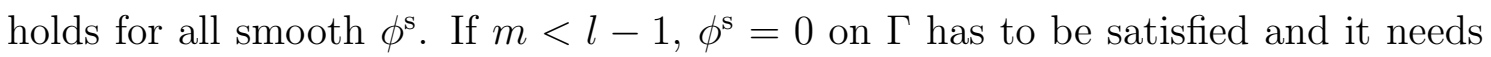
to be distinguished if $m=-1$ which gives

$$
\int_{S \times \Omega} \int_{\Gamma} a(x, y, t)\left(u_{1}^{\mathrm{p}}(x, y, t)+y \cdot \nabla_{x} u(x, t)\right) \phi^{\mathrm{p}}(x, y, t) \mathrm{d} \sigma_{y} \mathrm{~d} x \mathrm{~d} t
$$

as the limit and $m<-1$, which also lets the interfacial-exchange term vanish but requires $\phi^{\mathrm{p}}=0$ on $\Gamma$. Choosing $\phi^{0}=\phi^{\mathrm{s}}=0$ leaves

$$
\begin{aligned}
\int_{S \times \Omega} \int_{Z^{\mathrm{p}}} D^{\mathrm{p}}(x, y, t)\left[\nabla_{x} u(x, t)+\nabla_{y} u_{1}^{\mathrm{p}}(x, y, t)\right] \nabla_{y} \phi^{\mathrm{p}}(x, y, t) \mathrm{d} x \mathrm{~d} t \\
\quad=\sigma^{\mathrm{p}}(m+2) \int_{S \times \Omega} \int_{\Gamma}\left(u_{1}^{\mathrm{p}}(x, y, t)+y \cdot \nabla_{x} u(x, t)\right) \phi^{\mathrm{p}}(x, y, t) \mathrm{d} \sigma_{y} \mathrm{~d} x \mathrm{~d} t
\end{aligned}
$$

for all $\phi^{\mathrm{p}}$. Choosing $\phi^{\mathrm{p}}=0$, using the information just obtained and doing the usual calculation in the elliptic term, the limit problem reads

$$
\begin{aligned}
\int_{S \times \Omega} \partial_{t} u(x, t) \phi^{0}(x, t) \mathrm{d} x \mathrm{~d} t+\int_{S \times \Omega} P^{m, l}(x, t) & \nabla_{x} u(x, t) \nabla_{x} \phi_{0}(x, t) \mathrm{d} x \mathrm{~d} t \\
& =\int_{S \times \Omega} \int_{Y} f(x, y, t) \mathrm{d} y \phi^{0}(x, t) \mathrm{d} x \mathrm{~d} t .
\end{aligned}
$$

In the third case, i.e. $l \geq 2$, the limit problem is given by

$$
\begin{aligned}
& \int_{S \times \Omega} \int_{Z^{\mathrm{p}}} \partial_{t} u^{\mathrm{p}}(x, t) \mathrm{d} y \phi^{0}(x, t) \mathrm{d} x \mathrm{~d} t+\int_{S \times \Omega} \int_{Z^{\mathrm{s}}} \partial_{t} u^{\mathrm{s}}(x, y, t) \phi^{\mathrm{s}}(x, y, t) \mathrm{d} y \mathrm{~d} x \mathrm{~d} t \\
& +\int_{S \times \Omega} \int_{Z^{\mathrm{p}}} D^{\mathrm{p}}(x, y, t)\left[\nabla_{x} u^{\mathrm{p}}(x, t)+\nabla_{y} u_{1}^{\mathrm{p}}(x, y, t)\right]\left[\nabla_{x} \phi^{0}(x, t)+\nabla_{y} \phi^{\mathrm{p}}(x, y, t)\right] \mathrm{d} x \mathrm{~d} t \\
& +\theta(l) \int_{S \times \Omega} \int_{Z^{\mathrm{s}}} D^{\mathrm{s}}(x, y, t) \nabla_{y} u^{\mathrm{s}}(x, y, t) \nabla_{y} \phi^{\mathrm{s}}(x, y, t) \mathrm{d} y \mathrm{~d} x \mathrm{~d} t
\end{aligned}
$$




$$
\begin{aligned}
& +\lim _{\varepsilon \rightarrow 0} \varepsilon \int_{S \times \Gamma_{\varepsilon}} \varepsilon^{m-1} f_{\varepsilon}^{\mathrm{ex}}(x, t) \phi^{0}(x, t) \mathrm{d} \sigma_{x} \mathrm{~d} t \\
& +\lim _{\varepsilon \rightarrow 0} \varepsilon \int_{S \times \Gamma_{\varepsilon}} f_{\varepsilon}^{\mathrm{ex}}(x, t)\left[\varepsilon^{m} \phi^{\mathrm{p}}(x, x / \varepsilon, t)-\varepsilon^{m-1} \phi^{\mathrm{s}}(x, x / \varepsilon, t)\right] \mathrm{d} \sigma_{x} \mathrm{~d} t \\
& =\int_{S \times \Omega} \int_{Z^{\mathrm{p}}} f^{\mathrm{p}}(x, y, t) \mathrm{d} y \phi^{0}(x, t) \mathrm{d} x \mathrm{~d} t+\int_{S \times \Omega} \int_{Z^{\mathrm{s}}} f^{\mathrm{s}}(x, y, t) \phi^{\mathrm{s}}(x, y, t) \mathrm{d} y \mathrm{~d} x \mathrm{~d} t .
\end{aligned}
$$

Again, the limit of the interfacial-exchange term needs to be identified. For the term tested with $\phi^{0}$, we apply the boundary condition and assume that we can pass to the limit. Since $m<1$, the part tested with $\phi^{\text {s }}$ does not converge which is why $\phi^{\mathrm{s}}=0$ on $\Gamma$ needs to be demanded. The part tested with $\phi^{\mathrm{p}}$ converges to zero if $m>-1$, to

$$
\int_{S \times \Omega} \int_{\Gamma} a(x, y, t)\left(u_{1}^{\mathrm{p}}(x, y, t)+y \cdot \nabla u(x, t)\right) \phi^{\mathrm{p}}(x, y, t) \mathrm{d} \sigma_{y} \mathrm{~d} x \mathrm{~d} t
$$

if $m=-1$ and $\phi^{\mathrm{p}}=0$ on $\Gamma$ needs to be demanded if $m<-1$. Identifying the problem for $\phi^{0}=\phi^{\mathrm{s}}=0$ in the usual way, the limit problem reads

$$
\begin{aligned}
& \int_{S \times \Omega} \int_{Z_{\mathrm{p}}} \partial_{t} u^{\mathrm{p}}(x, t) \mathrm{d} y \phi^{0}(x, t) \mathrm{d} x \mathrm{~d} t+\int_{S \times \Omega} \int_{Z^{\mathrm{s}}} \partial_{t} u^{\mathrm{s}}(x, t) \phi^{\mathrm{s}}(x, y, t) \mathrm{d} y \mathrm{~d} x \mathrm{~d} t \\
& +\int_{S \times \Omega} P^{m, l}(x, t) \nabla_{x} u^{\mathrm{p}}(x, t) \nabla_{x} \phi^{0}(x, t) \mathrm{d} x \mathrm{~d} t \\
& +\theta(l) \int_{S \times \Omega} \int_{\Gamma} D^{\mathrm{s}}(x, y, t) \nabla_{y} u^{\mathrm{s}}(x, y, t) \cdot \nu^{\mathrm{s}} \mathrm{d} \sigma_{y} \phi^{0}(x, t) \mathrm{d} x \mathrm{~d} t \\
& +\theta(l) \int_{S \times \Omega} \int_{Z^{\mathrm{s}}} D^{\mathrm{s}}(x, y, t) \nabla_{y} u^{\mathrm{s}}(x, y, t) \nabla_{y} \phi^{\mathrm{s}}(x, y, t) \mathrm{d} y \mathrm{~d} x \mathrm{~d} t \\
& \quad=\int_{S \times \Omega} \int_{Z^{\mathrm{p}}} f^{\mathrm{p}}(x, y, t) \mathrm{d} y \phi^{0}(x, t) \mathrm{d} x \mathrm{~d} t+\int_{S \times \Omega} \int_{Z^{\mathrm{s}}} f^{\mathrm{s}}(x, y, t) \phi^{\mathrm{s}}(x, y, t) \mathrm{d} y \mathrm{~d} x \mathrm{~d} t .
\end{aligned}
$$

Choosing $\phi^{0}=0$,

$$
\begin{aligned}
& \int_{S \times \Omega} \int_{Z^{\mathrm{s}}} \partial_{t} u^{\mathrm{s}}(x, y, t) \phi^{\mathrm{s}}(x, y, t) \mathrm{d} y \mathrm{~d} x \mathrm{~d} t \\
& +\theta(l) \int_{S \times \Omega} \int_{Z^{\mathrm{s}}} D^{\mathrm{s}}(x, y, t) \nabla_{y} u^{\mathrm{s}}(x, y, t) \nabla_{y} \phi^{\mathrm{s}}(x, y, t) \mathrm{d} y \mathrm{~d} x \mathrm{~d} t \\
& \quad=\int_{S \times \Omega} \int_{Z^{\mathrm{s}}} f^{\mathrm{s}}(x, y, t) \phi^{\mathrm{s}}(x, y, t) \mathrm{d} y \mathrm{~d} x \mathrm{~d} t
\end{aligned}
$$

for all $\phi^{\mathrm{s}}$ with $\phi^{\mathrm{s}}=0$ on $\Gamma$ is obtained. Choosing $\phi^{\mathrm{s}}=0$,

$$
\begin{aligned}
\int_{S \times \Omega} \int_{Z^{\mathrm{p}}} \partial_{t} u^{\mathrm{p}}(x, t) \mathrm{d} y \phi^{0}(x, t) \mathrm{d} x \mathrm{~d} t+\int_{S \times \Omega} P^{l, m}(x, t) \nabla_{x} u^{\mathrm{p}}(x, t) \nabla_{x} \phi^{0}(x, t) \mathrm{d} x \mathrm{~d} t \\
=-\theta(l) \int_{S \times \Omega} \int_{\Gamma} D^{\mathrm{s}}(x, y, t) \nabla_{y} u^{\mathrm{s}}(x, y, t) \cdot \nu^{\mathrm{s}} \mathrm{d} \sigma_{y} \phi^{0}(x, t) \mathrm{d} x \mathrm{~d} t \\
+\int_{S \times \Omega} \int_{Z^{\mathrm{p}}} f^{\mathrm{p}}(x, y, t) \mathrm{d} y \phi^{0}(x, t) \mathrm{d} x \mathrm{~d} t
\end{aligned}
$$

for all smooth $\phi^{0}$ is obtained. 
We have thus proved the following theorem:

\section{Theorem 4.6}

The limit functions $u^{\mathrm{p}}$ and $u^{\mathrm{s}}$ associated with the sequences of solutions $u_{\varepsilon}^{\mathrm{p}}$ and $u_{\varepsilon}^{\mathrm{s}}$ of the microscopic problem (4) satisfy the macroscopic limit problems stated in $\S 2$.

\section{Acknowledgements}

We would like to thank W. Jäger from the University of Heidelberg and S. A. Meier from the University of Bremen for the fruitful discussions. Moreover, the first authors appreciates the financial support of the German National Academic Foundation.

\section{References}

[1] U. Hornung. Applications of the homogenization method to flow and transport in porous media. In X. Shutie, editor, Summer School on Flow and Transport in Porous Media, Beijing, China, 8-26 August 1988, pages 167-222. World Scientific, 1992.

[2] M. A. Peter. Coupled reation-diffusion processes and evolving microstructure: mathematical modelling and homongenisation. PhD thesis, University of Bremen, 2006. Also: Logos Verlag Berlin, 2007.

[3] M. A. Peter. Homogenisation in domains with evolving microstructure. $C$. $R$. Mécanique, 335(7):357-362, 2007.

[4] J.-L. Auriault and H. I. Ene. Macroscopic modelling of heat transfer in composites with interfacial thermal barrier. Int. J. Heat Mass Tranfer, 37(18):2885$2892,1994$.

[5] J.-F. Bloch and J.-L. Auriault. Heat transfer in nonsaturated porous media: modelling by homogenisation. Transp. Porous Med., 30:301-321, 1998.

[6] É. Canon, H. Bensmina, and P. Valentin. On the modelling of generalized Taylor-Aris dispersion in chromatographs via multiple scales expansions. Transp. Porous Med., 36:307-339, 1999.

[7] M. A. Peter and M. Böhm. Scalings in homogenisation of reaction, diffusion and interfacial exchange in a two-phase medium. In M. Fila, A. Handlovicova, K. Mikula, M. Medved, P. Quittner, and D. Sevcovic, editors, Proc. Equadiff-11, pages 369-376, 2005.

[8] T. Arbogast, J. Douglas Jr., and U. Hornung. Derivation of the double porosity model of single phase flow via homogenization theory. SIAM J. Math. Anal., 21(4):823-836, 1990.

[9] A. Bensoussan, J.-L. Lions, and G. Papanicolaou. Asymptotic analysis for periodic structures. North-Holland, 1978. 
[10] E. Sanchez-Palencia. Non-homogeneous media and vibration theory. Springer, 1980.

[11] G. I. Barenblatt, I. P. Zheltov, and I. N. Kochina. Basic concepts in the theory of seepage of homogeneous liquids in fissured rocks. J. Appl. Math. Mech., 24:1286-1303, 1960.

[12] H. I. Ene and D. Poliševski. Model of diffusion in partially fissured media. $Z$. angew. Math. Phys., 53:1052-1059, 2002.

[13] U. Hornung. Miscible displacement. In U. Hornung, editor, Homogenization and Porous Media, pages 129-146. Springer, 1997.

[14] U. Hornung and W. Jäger. Diffusion, convection, adsorption, and reaction of chemicals in porous media. J. Diff. Eq., 92:199-225, 1991.

[15] M. Peszyńska. Flow through fissured media. Mathematical analysis and numerical approach. PhD thesis, University of Augsburg, Germany, 1992.

[16] R. E. Showalter. Microstructure models of porous media. In U. Hornung, editor, Homogenization and Porous Media, pages 183-202. Springer, 1997.

[17] C. Conca, J. I. Diaz, and C. Timofte. Effective chemical processes in porous media. Math. Mod. Meth. Appl. Sci., 13(10):1437-1462, 2003.

[18] U. Hornung, W. Jäger, and A. Mikelić. Reactive transport through an array of cells with semi-permeable membranes. Math. Mod. Num. Anal., 28(1):59-94, 1994.

[19] L. Pankratov, A. Piatnitskii, and V. Rybalko. Homogenized model of reactiondiffusion in a porous medium. C. R. Mecanique, 331:253-258, 2003.

[20] É. Canon and J.-N. Pernin. Homogenization of diffusion in composite media with interfacial barrier. Rev. Roumaine Math. Pures Appl., 44(1):23-36, 1999.

[21] H. I. Ene. On the microstructure models of porous media. Rev. Roumaine Math. Pures Appl., 46(2-3):289-295, 2001.

[22] R. Lipton. Heat conduction in fine scale mixtures with interfacial contact resistance. SIAM J. Appl. Math., 58(1):55-72, 1998.

[23] S. Monsurrò. Homogenization of a two-component composite with interfacial thermal barrier. Adv. Math. Sci. Appl., 13(1):44-63, 2003.

[24] P. Donato and S. Monsurrò. Homogenization of two heat conductors with an interfacial contact barrier. Anal. Appl., 2(3):247-273, 2004.

[25] J.-N. Pernin. Diffusion in composite solid: threshold phenomenon and homogenization. Int. J. Eng. Science, 37:1597-1610, 1999.

[26] M. Panfilov. Macroscale models of flow through highly heterogeneous porous media. Kluwer Academic, 2000. 
[27] G. Nguetseng. A general convergence result for a functional related to the theory of homogenization. SIAM J. Math. Anal., 20(3):608-629, 1989.

[28] G. Allaire. Homogenization and two-scale convergence. SIAM J. Math. Anal., 23(6):1482-1518, 1992.

[29] G. Allaire, A. Damlamian, and U. Hornung. Two-scale convergence on periodic surfaces and applications. In A. P. Bourgeat, C. Carasso, S. Luckhaus, and A. Mikelic, editors, Proceedings of the international conference on mathematical modelling of flow through porous media, pages 15-25. World Scientific, 1995.

[30] M. Neuss-Radu. Some extensions of two-scale convergence. C. R. Acad. Sci. Paris, Ser. I, 322:899-904, 1996.

[31] G. Allaire and M. Briane. Multiscale convergence and reiterated homogenisation. Proc. Roy. Soc. Edinb., 126A:297-342, 1996.

[32] D. Lukkassen, G. Nguetseng, and P. Wall. Two-scale convergence. Int. J. Pure Appl. Math., 2(1):35-86, 2002.

[33] G. Allaire. Homogénéisation et convergence à deux échelles. Application à un problème de convection diffusion. C. R. Acad. Sci. Paris, Ser. I, 312:581-586, 1991.

[34] A. Holmbom. Homogenization of parabolic equations an alternative approach and some corrector-type results. Appl. Math., 42(5):321-343, 1997. 